\title{
Two-loop renormalization-group analysis of the Burgers-Kardar-Parisi-Zhang equation
}

\author{
Erwin Frey \\ Institut für Theoretische Physik, Physik-Department der Technischen Universität München, \\ James-Franck-Straße, D-85747 Garching, Germany \\ Uwe Claus Täuber \\ Lyman Laboratory of Physics, Harvard University, Cambridge, Massachusetts 02138
}

(Received 21 April 1994)

\begin{abstract}
A systematic analysis of the Burgers-Kardar-Parisi-Zhang equation in $d+1$ dimensions by dynamic renormalization-group theory is described. The fixed points and exponents are calculated to two-loop order. We use the dimensional regularization scheme, carefully keeping the full $d$ dependence originating from the angular parts of the loop integrals. For dimensions less than $d_{c}=2$ we find a strong-coupling fixed point, which diverges at $d=2$, indicating that there is nonperturbative strong-coupling behavior for all $d \geq 2$. At $d=1$ our method yields the identical fixed point as in the one-loop approximation, and the two-loop contributions to the scaling functions are nonsingular. For $d>2$ dimensions, there is no finite strong-coupling fixed point. In the framework of a $2+\epsilon$ expansion, we find the dynamic exponent corresponding to the unstable fixed point, which describes the nonequilibrium roughening transition, to be $z=2+O\left(\epsilon^{3}\right)$, in agreement with a recent scaling argument by Doty and Kosterlitz, Phys. Rev. Lett. 69, 1979 (1992). Similarly, our result for the correlation length exponent at the transition is $1 / \nu=\epsilon+O\left(\epsilon^{3}\right)$. For the smooth phase, some aspects of the crossover from Gaussian to critical behavior are discussed.
\end{abstract}

PACS number(s): 05.40.+j, 64.60.Ht, 05.70.Ln, 68.35.Fx

\section{INTRODUCTION}

In recent years there has been much theoretical interest in the dynamics of growing interfaces in random media $[1,2]$. This problem has received particular attention not only because of its technological importance and applications [2], but also as the simplest nontrivial example of dynamic scale invariance in a nonequilibrium system. Kardar, Parisi, and Zhang (KPZ) have proposed a simple nonlinear Langevin equation [3] which has become a widely accepted and by now well-known description of the macroscopic aspects of a certain class of growth processes. This equation is closely related to a large variety of other problems ranging from randomly stirred fluids [4] (Burgers equation), dissipative transport [5] (the driven-diffusion equation), flame-front propagation $[6,7]$ (Kuramoto-Sivashinski equation), polymer physics [8], the dynamics of a sine-Gordon chain [9], and the behavior of magnetic flux lines in superconductors [10].

Through a simple transformation the Burgers-KPZ equation can be mapped onto the statistical mechanics of directed polymers in a random medium $[8,11]$. This problem seems to embody many of the features of more complex random systems such as spin glasses [12].

Due to the simplicity of its form, accompanied by a stunning complexity of its behavior, it has gradually taken on the role of an "Ising model of nonequilibrium dynamics." Therefore, any advances in understanding the behavior of the Burgers-KPZ equation may have broad impacts in both the fields of nonequilibrium dynamics as well as disordered systems.
Assuming that a coarse-grained interface of a $d$ dimensional substrate may be described by a height function $h(\mathbf{x}, t)$ with $\mathbf{x} \in \mathbb{R}^{d}, \mathrm{KPZ}$ proposed that the following nonlinear Langevin equation [3]:

$$
\frac{\partial h}{\partial t}=\nu \nabla^{2} h+\frac{\lambda}{2}(\nabla h)^{2}+\eta
$$

governs the macroscopic (large-distance, long-time) dynamics of a stochastically growing interface. The first term in Eq. (1.1) represents the surface tension which prefers a smooth surface. The second term describes the tendency of the surface to locally grow normal to itself and is entirely nonequilibrium in origin. The last term is a Langevin noise to mimic the stochastic nature of any growth process. In Eq. (1.1), the average growth velocity has been subtracted so that the noise has zero mean, i.e., $\langle\eta(\mathbf{x}, t)\rangle=0$. In the simplest case, the stochasticity is then described by an uncorrelated Gaussian noise with the second moment

$$
\left\langle\eta(\mathbf{x}, t) \eta\left(\mathbf{x}^{\prime}, t^{\prime}\right)\right\rangle=2 D \delta^{d}\left(\mathbf{x}-\mathbf{x}^{\prime}\right) \delta\left(t-t^{\prime}\right),
$$

where $D$ characterizes the noise amplitude.

The shape of the steady-state surface profile is completely characterized by the steady-state distribution function $\mathcal{P}(h(\mathbf{x}, t))$. The leading (second) moment of $\mathcal{P}$ is the truncated two-point correlation function

$$
C(\mathbf{x}, t) \equiv\left\langle\left[h\left(\mathbf{x}_{0}+\mathbf{x}, t_{0}+t\right)-h\left(\mathbf{x}_{0}, t_{0}\right)\right]^{2}\right\rangle .
$$

In Eq. (1.3), the mean value of $h$ has been implicitly sub- 
tracted, i.e., we use a moving reference frame such that $\left\langle\partial_{t} h(x, t)\right\rangle=0$. Because the equation of motion (1.1) is scale invariant [3], the correlation function assumes the scaling form

$$
C(x, t)=x^{2 \chi} F\left(t / x^{z}\right)
$$

where $x \equiv|\mathbf{x}|$. In Eq. (1.4), the roughness exponent $\chi$ describes the scaling of the width of the interface and the dynamic exponent $z$ characterizes the spread in time of disturbances on the surface. In the limits $y=0$ and $y \rightarrow \infty$, the scaling function $F(y)$ becomes $A=$ const and $B y^{2 \chi / z}$ (with $B=$ const), respectively, thus yielding the usual asymptotic scaling form of the correlation function,

$$
\begin{aligned}
& C(x, t=0)=A x^{2 \chi}, \\
& C(x=0, t)=B t^{2 \chi / z} .
\end{aligned}
$$

In cases where the nonlinear term in Eq. (1.1) is not relevant, one recovers the linear equation of Edwards and Wilkinson [13]. There the exponents are known exactly, $\chi_{0}=(2-d) / 2$ and $z_{0}=2$, as expected from the ensuing simple diffusion equation.

In the more interesting case where the nonlinear term is important, one may derive the exponent identity

$$
\chi+z=2
$$

which follows from the invariance of (1.1) with respect to an infinitesimal tilting of the surface [3] $(h \rightarrow h+\mathbf{v} \cdot \mathbf{x}$, $\mathbf{x} \rightarrow \mathbf{x}-\lambda \mathbf{v} t)$. This invariance is known as "Galilean invariance" due to the corresponding invariance of the Burgers equation [4]. Therefore, there is at most one independent exponent to be determined. However, that last link needed to determine the exponents has proven to be quite elusive despite considerable effort.

In the special case of $d=1$, which is realized, for example, in the expansion of a domain boundary separating the two phases of a two-dimensional Ising model, Eq. (1.1) also satisfies a fluctuation-dissipation theorem (FDT). Then one can show [1] that the stationary distribution function is

$$
P_{\mathrm{st}}(h(x))=\exp \left\{-\frac{\nu}{2 D} \int d x\left(\frac{\partial h}{\partial x}\right)^{2}\right\} .
$$

Hence $\chi=1 / 2$, as if the nonlinearity were absent. Given the relevance of nonlinearity in $d=1$, one immediately obtains $z=3 / 2$ through the exponent identity Eq. (1.6).

For dimensions $d>2$ an interface described by the Burgers-KPZ equation is expected, on the basis of renormalization-group arguments $[3,4]$, to undergo a transition from an asymptotically smooth to a rough profile as the effective strength of the nonlinear coupling $\lambda^{2} D / \nu^{3}$ is increased. The exponents in the rough phase are only known from numerical simulations. The most recent values [14] seem to settle between the Wolf-Kertesz conjecture [15] $\chi / z=1 /(2 d+1)$ and the one by Kim and Kosterlitz [16] $\chi / z=1 /(d+2)$. Despite those results from numerical simulations, there has been very little progress towards an analytical theory which goes beyond the original paper [3], at least on the basis of (refined) perturbational approaches. The main obstacle within a systematic treatment originates from a renormalizationgroup (RG) flow towards a strong-coupling fixed point. Therefore, dynamic renormalization-group (DRG) methods have had very limited success. The main qualitative results obtained $[3,4]$ are (i) the interface is always rough (i.e., the nonlinearity is relevant) for $d \leq 2[17]$ and (ii) there is a dynamic phase transition between a smooth phase (nonlinearity irrelevant, $\chi=\chi_{0}, z=z_{0}$ ) and a rough phase where the nonlinearity is again relevant for $d>2$. Quantitatively, DRG provides a perturbative access to the dynamic phase transition point via a $d=2+\epsilon$ expansion. Unfortunately, DRG tells us virtually nothing about the most interesting aspect, namely the rough phase of the interface. The method breaks down because the DRG fixed point describing the rough phase is a strong-coupling fixed point generally not accessible by any perturbational means. This problem persists even in $d=1$, where the exponents themselves are already known exactly.

Recently, there has been some progress in understanding the behavior at the roughening transition itself, which is described by an unstable fixed point in the DRG theory. Doty and Kosterlitz [18] have argued that the dynamic exponent $z_{c}$ at the KPZ roughening transition equals 2 for all dimensions $d \geq 2$. Their argument is based on the mapping of the Burgers-KPZ equation to the equilibrium model of directed polymers in a random medium and a standard scaling argument usually applied for glassy systems, also very similar to the derivation of hyperscaling at critical points. Basically, they conclude that at a finite-temperature fixed point, one expects the scale of the free energy (which corresponds to the height in the Burgers-KPZ equation) to be given by the temperature, which is finite. This implies, for the roughness exponent, $\chi_{c}=0$ and $z_{c}=2$ via the exponent identity Eq. (1.6). This view is indeed supported by numerical simulations by Tang, Nattermann, and Forrest [19]. Based on the one-loop approximation, Nattermann and Tang [20] also discuss the interesting crossover scaling behavior of the Burgers-KPZ equation.

The purpose of this paper is to go beyond the oneloop perturbation theory, which is plagued by some artifacts, and perform a two-loop calculation, in order to possibly clarify which aspects are generally accessible to a DRG treatment and which are not, and also to establish certain trends, which might provide clues even beyond the two-loop approximation. We shall apply the field-theoretical version of the DRG in the formulation of Bausch, Janssen, and Wagner $[21,22]$. Using the dimensional regularization procedure [23] for a massless theory such as that provided by Eq. (1.1) requires a clear distinction between infrared and ultraviolet singularities, only the latter to be included in the renormalization constants. Also, in order to be able to correctly describe the exactly known behavior in $d=1$ dimensions, performing an $\epsilon$ expansion turns out to be a very delicate procedure. For $d<2$ our results indicate that such an expansion becomes inconsistent, the strong-coupling fixed point diverging for $d \rightarrow 2$, which is drastically different from 
the usual situations such as, e.g., in the paradigmatic $\phi^{4}$ theory $[24,25]$. On the other hand, for $d>2$ the now unstable fixed point describing the roughening transition may be treated in the framework of a $2+\epsilon$ expansion. The situation here can in fact be compared to the similar treatment of the $\mathrm{O}(n)$ nonlinear $\sigma$ model [26], where the nonlinear coupling corresponds to the transition temperature and is of order $\epsilon$ too.

During the process of completing this paper, we became aware of a similar field-theoretic two-loop analysis of the Burgers-KPZ equation by Sun and Plischke [27]. These authors, however, apply a somewhat different procedure and most of their results are clearly incompatible with ours, as shall be discussed below.

The outline of this paper is as follows. In Sec. II we establish a field-theoretic formulation for the nonlinear Langevin equation (1.1) and analyze the form of the propagators and vertices. Particular attention is paid to the symmetries of the Burgers-KPZ equation. We discuss the time-reversion symmetry and the existence of fluctuation-dissipation theorems due to detailed-balance properties of the underlying stochastic equation of motion. Furthermore, the Ward-Takahashi identities resulting from the Galilean invariance of the Burgers-KPZ equation are discussed. In Sec. III we proceed with a detailed description of our renormalization procedure and regularization scheme, elaborating especially on how we handle the $d$ dependences of various origins appearing in the singular terms. We then discuss the results of our two-loop perturbation theory, i.e., the behavior of Wilson's flow functions, the fixed points of the RG, and the resulting critical exponents as functions of the dimension $d$. We shall also investigate the scaling behavior at the dynamic roughening transition, as well as some features of the crossover from Gaussian to critical behavior in the flat phase. Finally, in Sec. IV we discuss our results and provide an outlook on future developments. In the Appendix we present some details of the two-loop calculation, listing the Feynman diagrams and analytical results for the two-point vertex functions, and the formulas for evaluating the integrals in the dimensional regularization scheme.

\section{MODEL EQUATIONS, PERTURBATION THEORY, AND WARD IDENTITIES}

In this section we establish a path integral formulation for the nonlinear Langevin equation Eq. (1.1), where particular attention is paid to the symmetries of the BurgersKPZ equation. Readers who are not familiar with these techniques may proceed to Sec. III A, where a formulation of the dynamic renormalization group is given which does not utilize the more formal methods described in this section. We note, however, that the formulation of dynamics in terms of path integrals provides a quite powerful tool which allows a systematic analysis of higher order terms as well as general theorems, such as fluctuationdisspation theorems and Ward-Takahashi identities.

\section{A. The model and dynamic functional}

We begin by reformulating the stochastic dynamics in terms of path integrals. Let us consider a coarse-grained interface of a $d$-dimensional substrate, described by a height function $h(\mathbf{x}, t)$ with $\mathbf{x} \in \mathbb{R}^{d}$, whose time evolution is governed by the Burgers-KPZ equation (the subscripts 0 will henceforth denote unrenormalized quantities),

$$
\frac{\partial h(\mathbf{x}, t)}{\partial t}=\nu_{0} \nabla^{2} h(\mathbf{x}, t)+\frac{\lambda_{0}}{2}(\nabla h(\mathbf{x}, t))^{2}+\eta(\mathbf{x}, t) .
$$

The random forces $\eta(\mathbf{x}, t)$ can be taken to be Gaussian distributed,

$$
W[\eta] \propto \exp \left\{-\frac{1}{4 D_{0}} \int d^{d} x \int d t \eta(\mathbf{x}, t)^{2}\right\}
$$

with mean zero and short-ranged spatial and temporal correlations

$$
\begin{aligned}
& \langle\eta(\mathbf{x}, t)\rangle=0 \\
& \left\langle\eta(\mathbf{x}, t) \eta\left(\mathbf{x}^{\prime}, t^{\prime}\right)\right\rangle=2 D_{0} \delta^{(d)}\left(\mathbf{x}-\mathbf{x}^{\prime}\right) \delta\left(t-t^{\prime}\right) .
\end{aligned}
$$

From the standard dynamic field theory formulation $[21,22,28,29]$ of Langevin dynamics, upon averaging over the noise distribution and introducing auxiliary response fields $\tilde{h}(\mathbf{x}, t)$, one can turn the stochastic differential equation Eq. (2.1) into a "free energy" functional (generating functional)

$$
\begin{aligned}
Z[j, \tilde{j}]= & \int \mathcal{D}[h] \mathcal{D}[i \tilde{h}] \exp \{\mathcal{J}[\tilde{h}, h] \\
& \left.+\int d^{d} x \int d t[\tilde{j} \tilde{h}+j h]\right\}
\end{aligned}
$$

with the Janssen-De Dominics functional given by

$$
\begin{aligned}
\mathcal{J}[\tilde{h}, h]= & \int d^{d} x \int d t\left\{D_{0} \tilde{h} \tilde{h}\right. \\
& \left.-\tilde{h}\left[\frac{\partial h}{\partial t}-\nu_{0} \nabla^{2} h-\frac{\lambda_{0}}{2}(\nabla h)^{2}\right]\right\} .
\end{aligned}
$$

Correlation and response functions can now be expressed as functional averages with weight $\exp \{\mathcal{J}[\tilde{h}, h]\}$.

\section{B. Perturbation theory}

The propagators and correlators are determined by the harmonic ("Gaussian") part

$$
\begin{aligned}
\mathcal{J}_{0}[\tilde{h}, h] & =\int_{\mathbf{k}} \int_{\omega} \tilde{h}(\vec{k})\left\{D_{0} \tilde{h}(-\vec{k})-\left[i \omega+\nu_{0} k^{2}\right] h(-\vec{k})\right\} \\
& =-\frac{1}{2} \int_{\mathbf{k}} \int_{\omega}(\tilde{h}(\vec{k}), h(\vec{k})) \mathbf{A}(\vec{k})\left(\begin{array}{c}
\tilde{h}(-\vec{k}) \\
h(-\vec{k})
\end{array}\right)
\end{aligned}
$$


of the dynamic functional, with

$$
\mathbf{A}(\vec{k})=\left(\begin{array}{cc}
-2 D_{0} & i \omega+\nu_{0} k^{2} \\
-i \omega+\nu_{0} k^{2} & 0
\end{array}\right)
$$

Here we have introduced $\vec{k}=(\mathbf{k}, \omega)$ and defined the Fourier-transformed quantities by

$$
h(\mathbf{x}, t)=\int_{\mathbf{q}} \int_{\omega} h(\mathbf{q}, \omega) e^{i(\mathbf{q} \mathbf{x}-\omega t)},
$$

using the abbreviations $\int_{\mathbf{q}} \cdots=(2 \pi)^{-d} \int d^{d} q \cdots$ and $\int_{\omega} \cdots=(2 \pi)^{-1} \int d \omega \cdots$. The interaction part $\mathcal{J}_{\text {int }}$ is proportional to the coupling constant $\lambda_{0}$ and given by

$$
\begin{aligned}
\mathcal{J}_{\text {int }}[\tilde{h}, h]=i^{2} \frac{\lambda_{0}}{2} \int_{\left\{\mathbf{k}_{i}\right\}} & \left(\mathbf{k}_{2} \cdot \mathbf{k}_{3}\right) \tilde{h}\left(\vec{k}_{1}\right) h\left(\vec{k}_{2}\right) h\left(\vec{k}_{3}\right) \\
& \times \delta\left(\sum \vec{k}_{i}\right)
\end{aligned}
$$

The corresponding diagrammatic representation of the response and correlation propagators and the vertex is shown in Fig. 1. With the above perturbation theory at hand we can now calculate the cumulants \langle\rangle$_{c}$ of the correlation and response functions defined by functional derivatives of $F[\tilde{j}, j]=\ln Z[\tilde{j}, j]$ with respect to the sources $\tilde{j}$ and $j$, respectively:

$$
\begin{aligned}
G_{\tilde{N}, N}\left(\vec{k}_{1} ; \ldots ; \vec{k}_{\tilde{N}} ; \vec{k}_{\tilde{N}+1} ; \ldots ; \vec{k}_{\tilde{N}+N}\right) \\
=\left\langle\tilde{h}\left(\vec{k}_{1}\right) \cdots \tilde{h}\left(\vec{k}_{\tilde{N}}\right) h\left(\vec{k}_{\tilde{N}+1}\right) \cdots h\left(\vec{k}_{\tilde{N}+N}\right)\right\rangle_{c} \\
=\left.\frac{\delta^{\tilde{N}+N} \ln Z\left[\tilde{j}_{, j]}\right.}{\delta \tilde{j}\left(-\vec{k}_{1}\right) \cdots \delta j\left(-\vec{k}_{\tilde{N}+N}\right)}\right|_{\tilde{j}, j=0} \cdot \quad(2.11)
\end{aligned}
$$

It is convenient to consider the vertex functions $\Gamma_{\tilde{N}, N}$, which can be obtained from the cumulants by a Legendre transformation,

$$
\Gamma[\tilde{h}, h]=-F[\tilde{j}, j]+\int d^{d} x \int d t(\tilde{h} \tilde{j}+h j),
$$

where

$$
h=\frac{\delta F}{\delta j}, \quad \tilde{h}=\frac{\delta F}{\delta \tilde{j}} .
$$

In equilibrium dynamics the linear response is defined by adding to the Hamiltonian an external field which couples linearly to the field $h$. For nonequilibrium random processes, however, the stationary probability distribution function (which is the analog of the Gibbsian measure) is not known a priori, except for certain cases where the

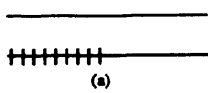

(a)

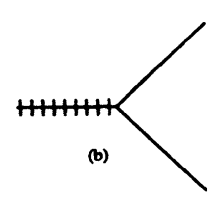

FIG. 1. Basic elements of the dynamical perturbation theory for the Burgers-Kardar-Parisi-Zhang equation. (a) Correlation and response propagators. (b) Three-point vertex. potential conditions (see below) are fulfilled and the random process obeys a detailed-balance condition [30,31]. Therefore, we define the linear response in terms of (deterministic) "external" fields $b$ added linearly to the drift term in the equation of motion

$$
V(h) \rightarrow V(h)+b .
$$

This external field $b$ corresponds to the source $\tilde{j}$ in the generating functional. This remark should also clarify why $\tilde{h}$ is called a "response field" (see Ref. [22]).

We proceed with a simple dimensional analysis to determine the engineering dimensions of the parameters and fields. Since the dynamic functional has to be dimensionless $[\mathcal{J}]=1$, upon defining inverse length and time scales according to $[q]=\Lambda$ and $[\omega]=\nu_{0} \Lambda^{2}$, respectively, we arrive at the following "naive" dimensions

$$
\begin{aligned}
& {[h]=\Lambda^{-d / 2-3} \nu_{0}^{-3 / 2} D_{0}^{1 / 2},} \\
& {[\tilde{h}]=\Lambda^{-d / 2-1} \nu_{0}^{-1 / 2} D_{0}^{-1 / 2},} \\
& {\left[\lambda_{0}\right]=\Lambda^{-d / 2+1} \nu_{0}^{-3 / 2} D_{0}^{-1 / 2} .}
\end{aligned}
$$

Hence the engineering dimension of the effective coupling constant is $\left[\lambda_{0}^{2} D_{0} / \nu_{0}^{3}\right]=\Lambda^{2-d}$. The space dimension at which the effective coupling constant becomes dimensionless is $d_{c}=2$. In simple cases such as a $\phi^{4}$ theory this is the dimension which separates Gaussian from critical behavior. However, this simple scenario is not at all what one finds for the Burgers-KPZ equation. As we will see in the following, $d_{c}=2$ is the dimension below which the behavior of the interface fluctuations are described by a finite "strong-coupling" fixed point, which is not of order $\epsilon=d-2$, but rather of order $O(1)$. Above $d_{c}=2$, on the other hand, the two-loop calculation yields an infrared unstable fixed point which is of order $O(\epsilon)$. For effective coupling constants less than this unstable fixed point, the RG flow tends to small coupling, i.e., the interface fluctuations are described by a capillary wave Hamiltonian $\mathcal{H} \propto \int d^{d} x(\nabla h)^{2}$ (corresponding to Gaussian behavior). When the coupling constant becomes larger than the value of the unstable fixed point, the RG flow tends to infinity. The corresponding strong-coupling behavior is hence described by a strong-coupling fixed point which is obviously not accessible by perturbative methods (of any finite order in perturbation theory). Resummation techniques such as the quite successful mode-coupling approach [32] are needed. Those methods have been applied to the Burgers-KPZ problem in Refs. [5,33-38]. They allow a determination of the critical exponents and the scaling functions. However, little is known to date about whether or in what sense those self-consistency methods constitute a controlled expansion with some possibly small parameter. The two-loop calculation in this paper provides some hints on the validity of the mode-coupling theory in the $(1+1)$-dimensional case (see Sec. III).

\section{Ward-Takahashi identities}

As usual, internal symmetries of the effective Lagrangian, i.e., here the Langevin equation, are important 
in determining the number of independent renormalization factors of the theory. The most important symmetry of the Burgers-KPZ equation is its behavior with respect to tilting of interface by an (infinitesimal) angle $\mathbf{v}$ (this corresponds to the Galilean invariance of Burgers's equation in hydrodynamics),

$$
\begin{aligned}
& h^{\prime}(\mathbf{x}, t)=h\left(\mathbf{x}+\lambda_{0} \mathbf{v} t, t\right)+\mathbf{v} \cdot \mathbf{x}, \\
& \tilde{h}^{\prime}(\mathbf{x}, t)=\tilde{h}\left(\mathbf{x}+\lambda_{0} \mathbf{v} t, t\right)
\end{aligned}
$$

In Fourier space the latter equations read

$$
\begin{aligned}
& h^{\prime}(\mathbf{q}, t)=e^{i \lambda_{0} \mathbf{v} \cdot \mathbf{q} t} h(\mathbf{q}, t)-i \mathbf{v} \cdot \frac{\partial}{\partial \mathbf{q}} \delta(\mathbf{q}), \\
& \tilde{h}^{\prime}(\mathbf{q}, t)=e^{i \lambda_{0} \mathbf{v} \cdot \mathbf{q} t} \tilde{h}(\mathbf{q}, t) .
\end{aligned}
$$

The invariance of the generating functional of the vertex functions under the above transformations implies the following Ward-Takahashi identity:

$$
\begin{aligned}
\delta \Gamma= & \mathbf{v} \cdot \int_{\mathbf{q}} \int d t\left[i \lambda_{0} \mathbf{q} t\left(\frac{\delta \Gamma}{\delta h(\mathbf{q}, t)} h(\mathbf{q}, t)+\frac{\delta \Gamma}{\delta \tilde{h}(\mathbf{q}, t)} \tilde{h}(\mathbf{q}, t)\right)\right. \\
& \left.+i \delta(\mathbf{q}) \frac{\partial}{\partial \mathbf{q}} \frac{\delta \Gamma}{\delta h(\mathbf{q}, t)}\right]=0 .
\end{aligned}
$$

Functional derivatives with respect to $\tilde{h}\left(\mathbf{q}^{\prime}, t^{\prime}\right)$ and $h\left(\mathbf{q}^{\prime \prime}, t^{\prime \prime}\right), \tilde{h}\left(\mathbf{q}^{\prime \prime}, t^{\prime \prime}\right)$ yield relations between three-point and two-point vertex functions

$$
\begin{aligned}
\lambda_{0}\left(\mathbf{q}^{\prime} t^{\prime}+\right. & \left.\mathbf{q}^{\prime \prime} t^{\prime \prime}\right) \Gamma_{\tilde{h} h}\left(\mathbf{q}^{\prime}, t^{\prime} ; \mathbf{q}^{\prime \prime}, t^{\prime \prime}\right) \\
& =-\left.\frac{\partial}{\partial \mathbf{q}} \int d t \Gamma_{\tilde{h} h h}\left(\mathbf{q}^{\prime}, t^{\prime} ; \mathbf{q}^{\prime \prime}, t^{\prime \prime} ; \mathbf{q}, t\right)\right|_{\mathbf{q}=0},
\end{aligned}
$$

$\lambda_{0}\left(\mathbf{q}^{\prime} t^{\prime}+\mathbf{q}^{\prime \prime} t^{\prime \prime}\right) \Gamma_{\tilde{h} \tilde{h}}\left(\mathbf{q}^{\prime}, t^{\prime} ; \mathbf{q}^{\prime \prime}, t^{\prime \prime}\right)$

$$
=-\left.\frac{\partial}{\partial \mathbf{q}} \int d t \Gamma_{\tilde{h} \tilde{h} h}\left(\mathbf{q}^{\prime}, t^{\prime} ; \mathbf{q}^{\prime \prime}, t^{\prime \prime} ; \mathbf{q}, t\right)\right|_{\mathbf{q}=0},
$$

or, in frequency representation,

$$
\begin{aligned}
i \lambda_{0}(\mathbf{q} \cdot \mathbf{p}) \frac{\partial}{\partial \omega} \Gamma_{\tilde{h} h} & (\mathbf{q}, \omega ;-\mathbf{q},-\omega) \\
& =\Gamma_{\tilde{h} h h}(\mathbf{q}-\mathbf{p}, \omega ;-\mathbf{q},-\omega ; \mathbf{p}, 0),
\end{aligned}
$$

$i \lambda_{0}(\mathbf{q} \cdot \mathbf{p}) \frac{\partial}{\partial \omega} \Gamma_{\tilde{h} \tilde{h}}(\mathbf{q}, \omega ;-\mathbf{q},-\omega)$

$$
=\Gamma_{\tilde{h} \tilde{h} h}(\mathbf{q}-\mathbf{p}, \omega ;-\mathbf{q},-\omega ; \mathbf{p}, 0) .
$$

From the diffusive dynamics and the corresponding $\mathbf{q}$ dependence of the vertices follows the exact result (valid to any order in perturbation theory)

$$
\Gamma_{\tilde{h} h}(\mathbf{0}, \omega)=i \omega
$$

Hence the fields $\tilde{h}$ and $h$ do not renormalize [39]. The Ward identities Eqs. (2.25) and (2.26) then yield immediately that there is also no renormalization of the nonlinearity $\lambda$. Therefore we shall henceforth drop the index 0 for $\lambda$, previously denoting the unrenormalized quantity, as we have already anticipatingly done for the fields.

\section{RENORMALIZATION-GROUP THEORY}

In this section we present our results from the renormalization group analysis of the Burgers-KPZ equation to two-loop order. We focus on the main concepts and results. For more details we refer the reader to the Appendix, where some intermediate results for the calculations of the vertex functions and the renormalization constants are presented.

\section{A. General considerations}

By now several powerful methods have evolved, and are frequently used, by which dynamical critical phenomena are analyzed. The method by which the Burgers$\mathrm{KPZ}$ equation has been investigated first [4] is based on the dynamic renormalization group as formulated by Ma and Mazenko [40]. This scheme is in close analogy to Wilson's momentum-shell procedure [41] and has the appealing feature of being conceptually transparent and more "physical" than field-theoretic methods, which utilize a mapping of the stochastic equations of motion onto a dynamical functional $[29,28,21,22]$, and concepts originally developed for understanding quantum field theories $[24,25]$. The latter schemes have the advantage of providing a powerful tool for calculating correlation functions and interconnections between them in a systematic way, which can be crucial if one intends to go beyond one-loop order.

Since the two-loop calculations will become quite complicated, and there is certainly the danger that the important physical and conceptual points may be obscured by the tedious calculations, we regard it as useful at this point to review the dynamic renormalization-group procedure applying both Wilson's scheme and field-theoretic methods to one-loop approximation. In order to keep our arguments as simple as possible, we also refrain from using the mapping onto a dynamic functional in this chapter and formulate the theoretical concepts by using just the equation of motion.

Equation (1.1) reads in Fourier space

$$
\begin{aligned}
h(\vec{q})= & G_{0}(\vec{q}) \eta(\vec{q}) \\
& +\frac{1}{2} G_{0}(\vec{q}) \int_{\vec{k}} V_{0}\left(\mathbf{q}_{+} ; \mathbf{q}_{-}\right) h\left(\vec{q}_{+}\right) h\left(\vec{q}_{-}\right) .
\end{aligned}
$$

In Eq. (3.1) $G_{0}(\mathbf{q}, \omega)=1 /\left(\nu_{0} \mathbf{q}^{2}-i \omega\right)$ denotes the "bare propagator," $V_{0}\left(\mathbf{q}_{1} ; \mathbf{q}_{2}\right)=-\lambda_{0} \mathbf{q}_{1} \cdot \mathbf{q}_{2}$ is the "bare vertex," and $\mathbf{q}_{ \pm} \equiv \mathbf{q} / 2 \pm \mathbf{k}, \omega_{ \pm} \equiv \omega / 2 \pm \nu$. For $V_{0}=0$, Eq. (3.1) is just the linear diffusion equation. For $V_{0} \neq 0$, the solution of Eq. (3.1) may be obtained iteratively by a perturbation expansion in powers of $V_{0}$. For exam- 
ple, the lowest-order correction to the response function $G=\delta\langle h\rangle / \delta \eta$ is

$$
G_{1}(\vec{q})=G_{0}(\vec{q})+G_{0}(\vec{q}) \Sigma_{1}(\vec{q}) G_{0}(\vec{q}),
$$

where $C_{0}(\mathbf{q})=2 D_{0}\left|G_{0}(\mathbf{q})\right|^{2}$ is the "bare correlator," and

$$
\Sigma_{1}(\vec{q})=-\int_{\vec{k}} V_{0}\left(\mathbf{q}_{+} ; \mathbf{q}_{-}\right) G_{0}\left(\vec{q}_{-}\right) C_{0}\left(\vec{q}_{+}\right) V_{0}\left(\mathbf{q}_{+} ; \mathbf{q}\right)
$$

represents the one-loop renormalization of the "selfenergy." Similarly, the lowest-order correction to the correlation function is

$$
C_{1}(\vec{q})=2 D_{1}(\vec{q})\left|G_{0}(\vec{q})\right|^{2},
$$

where

$$
D_{1}(\vec{q})=D_{0}+\frac{1}{4} \int_{\vec{k}} V_{0}\left(\mathbf{q}_{+} ; \mathbf{q}_{-}\right) C_{0}\left(\vec{q}_{-}\right) C_{0}\left(\vec{q}_{+}\right) V_{0}\left(\mathbf{q}_{+} ; \mathbf{q}_{-}\right)
$$

is the one-loop renormalization of the "noise spectrum." Higher loop orders could in principle be obtained from iteration of the equation of motion Eq. (3.1). But, since we are mainly interested in the conceptual problems now, we restrict ourselves for the sake of simplicity to the one-loop approximation. (The two-loop contributions and their corresponding diagrammatic representations are presented in Appendix A 1 and will be discussed in Sec. III B.) After performing the internal frequency integrals one obtains for the response and correlation functions at zero frequency $\omega=0$ and in the limit $\mathbf{q} \rightarrow \mathbf{0}$, respectively, the following results:

$$
\begin{aligned}
C_{1}(\mathbf{0}, 0)= & C_{0}(\mathbf{0}, 0) \\
& +2 \frac{\lambda^{2} D_{0}}{4 \nu_{0}^{3}} G_{0}(\mathbf{0}, 0)^{2} \int \frac{d^{d} k}{(2 \pi)^{d}} \frac{1}{k^{2}}+O\left(q^{2}\right), \\
G_{1}(\mathbf{q}, 0)= & G_{0}(\mathbf{q}, 0)-\frac{\lambda^{2} D_{0}}{4 \nu_{0}^{3}} G_{0}(\mathbf{q}, 0)^{2} \frac{2-d}{d} q^{2} \\
& \times \int \frac{d^{d} k}{(2 \pi)^{d}} \frac{1}{k^{2}}+O\left(q^{4}\right),
\end{aligned}
$$

where only the terms of lowest order in $q^{2}$ have been retained. It is essential to note that the prefactor $2-d$ in Eq. (3.7) is a geometry factor, stemming from the scalar products in the integrand, i.e., we have made use of the identity $\int_{\mathbf{k}}(\mathbf{q} \cdot \mathbf{k}) f(k)=\left(q^{2} / d\right) \int_{\mathbf{k}} k^{2} f(k)$ with $k=|\mathbf{k}|$. This $d$-dependent prefactor has nothing at all to do with the problems in perturbation theory, resulting from infrared divergences, to be discussed next. Namely, the naive perturbation theory breaks down due to the fact that the integral $\int_{k} k^{-2}$ in Eqs. (3.6) and (3.7) is manifestly infrared (IR) divergent at the lower cutoff. These divergences are of real physical origin and it is exactly those, by which one is lead to the introduction of the renormalization-group concept.

In Wilson's momentum space procedure [41] the RG transformation is defined as follows.

(1) Elimination. First, one eliminates those modes within a small momentum shell $k \in[\Lambda / b, \Lambda]$, where $b=e^{l}>1$. This results in an effective (renormalized) propagator for the remaining modes and allows the identification of an effective surface tension (diffusion constant) to one-loop order $[4,3]$

$$
\nu_{\mathrm{W}}=\nu_{0}\left[1+\frac{\lambda^{2} D_{0}}{4 \nu_{0}^{3}}\left(\frac{2-d}{d}\right) K_{d} \Lambda^{d-2} l\right],
$$

where $K_{d}=S_{d} /(2 \pi)^{d}$ and $S_{d}$ is the surface area of the unit sphere in $d$ dimensions. In an analogous way one finds $[4,3]$

$$
D_{\mathrm{W}}=D_{0}\left[1+\frac{\lambda^{2} D_{0}}{4 \nu_{0}^{3}}\left(\frac{1}{d}\right) K_{d} \Lambda^{d-2} l\right] .
$$

Note that the expressions (3.8) and (3.9) coincide in $d=$ 1 , as is required by the additional FDT valid only at this specific dimension. Without loss of generality we may set the cutoff $\Lambda$ to unity.

(2) Rescaling. After this elimination step has been performed, the resulting model has a cutoff which is reduced by a factor $b=e^{l}>1$. In order to remove this difference and arrive at a form more closely resembling the original system, the momenta, times, and height variable are rescaled according to $k \rightarrow k e^{-l}, t \rightarrow e^{z l} t$, and $h \rightarrow e^{\chi l} h$, respectively. Upon requiring that the equation of motion stays invariant under this scale transformation, the parameters and coupling constants transform as follows:

$$
\begin{aligned}
\nu_{0} & \rightarrow b^{z-2} \nu_{0}, \\
D_{0} & \rightarrow b^{-d-2 \chi+z} D_{0}, \\
\lambda_{0} & \rightarrow b^{\chi+z-2} \lambda_{0} .
\end{aligned}
$$

Combining these contributions results in differential recursion relations (for infinitesimal RG transformations where $l \ll 1)[4,3,20]$ :

$$
\begin{aligned}
\frac{d \nu_{\mathrm{W}}}{d l} & =\nu_{\mathrm{W}}\left[z-2+K_{d} \frac{\lambda_{\mathrm{W}}^{2} D_{\mathrm{W}}}{4 \nu_{\mathrm{W}}^{3}}\left(\frac{2-d}{d}\right)\right], \\
\frac{d D_{\mathrm{W}}}{d l} & =D_{\mathrm{W}}\left[z-2 \chi-d+K_{d} \frac{\lambda_{\mathrm{W}}^{2} D_{\mathrm{W}}}{4 \nu_{\mathrm{W}}^{3}}\right], \\
\frac{d \lambda_{\mathrm{W}}}{d l} & =\lambda_{\mathrm{W}}[\chi+z-2],
\end{aligned}
$$

where the suffix $\mathrm{W}$ indicates that the running coupling constants and parameters are obtained within the framework of Wilson's momentum shell method. (We shall later introduce another set of flow functions in the context of renormalized field theory.) Note that there is no perturbative correction to the flow of $\lambda_{\mathrm{W}}$, which can be understood as a direct consequence of the WardTakahashi identities (2.23)-(2.26).

(3) Fixed point and critical exponents. The last step is to determine the exponents $z$ and $\chi$ such that the parameters $\nu_{\mathrm{W}}$ and $D_{\mathrm{W}}$ are not changed, for we have then obviously arrived at a scale-invariant situation. We define an effective coupling constant by

$$
g_{\mathrm{W}}=K_{d} \frac{\lambda_{\mathrm{W}}^{2} D_{\mathrm{W}}}{4 \nu_{\mathrm{W}}^{3}}
$$

and obtain, for the corresponding flow, 


$$
\frac{d g_{\mathrm{W}}(l)}{d l}=(2-d) g_{\mathrm{W}}(l)+\frac{2(2 d-3)}{d} g_{\mathrm{W}}^{2}(l)+O\left(g_{\mathrm{W}}^{3}\right) .
$$

From these recursion relations one can solve for the RG fixed points, i.e., points in parameter space which are invariant under scale transformations. Thus setting $d g_{\mathrm{W}}(l) / d l=0$ in Eq. (3.17) yields the Gaussian fixed point $g_{0}^{*}=0$ and also one nontrivial fixed point

$$
g_{1}^{*}=\frac{d(d-2)}{2(2 d-3)},
$$

which is positive for $0 \leq d<3 / 2$, diverges at $d=3 / 2$, then rises again from $-\infty$, crossing the zero axis at $d=2$, and becoming positive for $d>2$ (Fig. 2). In the physical region, $g>0, g_{1}^{*}$ turns out to be stable ("attractive") for $d<3 / 2$, thus providing nontrivial (strong-coupling) scaling behavior, while for $d>2$ the Gaussian fixed point is stable. Hence, depending on its initial value, $g_{\mathrm{W}}(l)$ will either flow to $g_{0}^{*}$ or to the strong-coupling "fixed point" $g_{\mathrm{W}} \rightarrow \infty$. Thus for $d>2$ a dynamic phase transition ("roughening transition") is found, governed by the unstable fixed point $g_{1}^{*}$. The fact that there is no finite positive fixed point in the range $3 / 2<d<2$ will turn out to be an artifact of the one-loop approximation (see Sec. III B).

Upon setting $d \nu_{\mathrm{W}} / d l=0, d D_{\mathrm{W}} / d l=0$, and $g_{\mathrm{W}}=g^{*}$, one finds $z_{0}=2$ and $\chi_{0}=(2-d) / 2$ at the Gaussian fixed point, while at any nontrivial fixed point $g^{*} \neq 0$ the critical exponents become

$$
\begin{aligned}
& z=2+\left(\frac{d-2}{d}\right) g^{*}, \\
& \chi=-\left(\frac{d-2}{d}\right) g^{*} .
\end{aligned}
$$

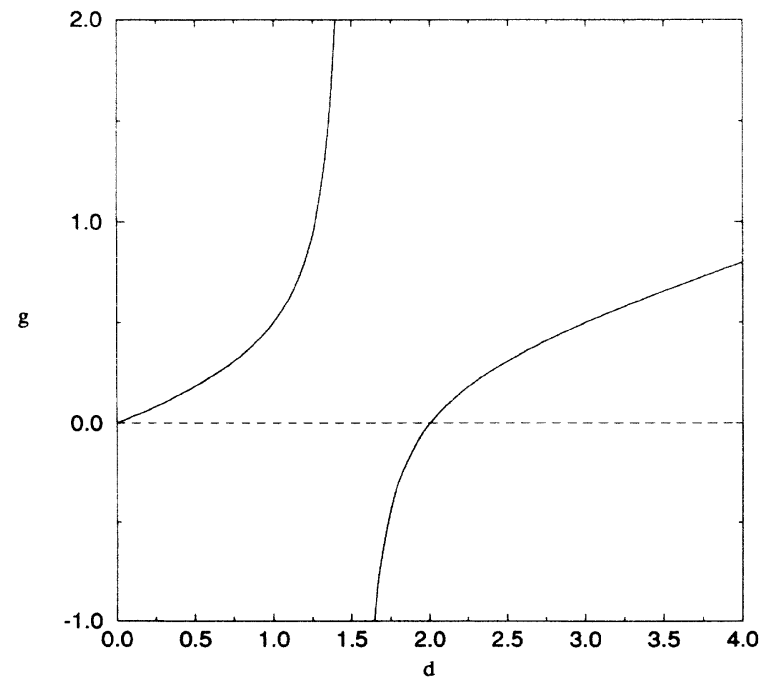

FIG. 2. One-loop fixed point of the Burgers-KPZ equation as a function of the surface dimension $d$. The divergence at $d=3 / 2$ turns out to be an artifact of the one-loop approximation.
Note that Eq. (3.15) forces $z+\chi=2$, if $\lambda \neq 0$. Thus the exponent identity (1.6) holds for any finite fixed point, which implies that knowledge of either the flow of $\nu_{\mathrm{W}}$ or $D_{\mathrm{W}}$ suffices in order to determine the critical exponents in its vicinity.

(As already mentioned, the flow obtained from these one-loop equations still contains some artifacts, which are due to the low order of perturbation theory considered. Yet our concern in this section is a comparison of the various methods available to determine the RG flow of the parameters and couplings rather than a detailed description of the physical behavior.)

Let us now compare these results with the various fieldtheoretic techniques using different regularization procedures. In field theory, the cutoff $\Lambda$ is set to infinity, thus leading to ultraviolet (UV) divergences above a certain critical dimension $d_{c}$ (in our case, $d_{c}=2$ ). There are basically two regularization methods, by which these integrals are assigned meaningful values, that are frequently used in field theories: (i) reintroducing the cutoff and (ii) dimensional regularization. In the former case the original UV singularities appear as logarithms or powers of $\Lambda$, in the latter as $1 /\left(d_{c}-d\right)$ poles. The criteria of choosing either of them are mostly guided by convenience, i.e., the amount of effort by which the corresponding integrals may be evaluated. Using those more formal field-theoretic methods one has to be careful, however, especially if one deals with massless theories, as is the case here. Let us explain why some specific attention is required (see also Ref. [25], p. 161). In dimensional regularization $[23,24]$ one has

$$
\int \frac{d^{d} p}{p^{m}}=0
$$

which is a (nontrivial for even $m$ ) consequence of the dilatation property of the integrals defined here by dimensional continuation. This result can be regarded as a cancellation between infrared (IR) and ultraviolet (UV) divergences. This often convenient property of integrals within the dimensional regularization scheme may, however, have quite dangerous consequences: In a field theory involving massless fields (for which the Burgers$\mathrm{KPZ}$ equation is an important example), the latter generate IR singularities, which, again, have the signature of $1 /\left(d_{c}-d\right)$ poles. Thus, contrary to calculations using a finite cutoff, the dimensional regularization scheme is in danger of mixing UV and IR poles, as is obvious from Eq. (3.21). It is, however, crucial to clearly separate the $U V$ divergences and avoid any mixing with the $I R$ singularities, as will be explained below. This really may be the source of many mistakes if this method is applied without sufficient precaution. Thus it is essential to employ an IR cutoff in the integrals. In the case of the Burgers-KPZ equation, we cannot simply introduce a mass term, however, because this would immediately violate the Galilean invariance discussed in Sec. II C. Therefore we have to evaluate all quantities explicitly at finite external wave vectors or frequencies. For the calculations in Appendix A 2, we have chosen the convenient normalization point (NP): $\mathbf{q}=\mathbf{0}, i \omega / 2 \nu=\mu^{2}$. 
In order to make our arguments clear we want to very briefly review some of the fundamental ideas behind renormalized field theory and the connection to critical phenomena $[24,25,42]$. From the dimensional engineering in Sec. II and simple power counting arguments, it follows that perturbation theory fails to describe the critical theory below the critical dimension $d_{c}$ because of IR divergences (see above). Above $d_{c}$, on the other hand, the integrals become UV divergent. These UV singularities may be handled very effectively by using the wellestablished technology of quantum field theory, namely, by introducing renormalization constants into which the UV poles are absorbed. From these the Callan-Symanzik equations can be inferred, which describe the dependence of the renormalized quantities on the renormalization scale. Already by a heuristic argument (see Ref. [42], p. 271), the intimate relation of the IR and UV singularities may be explained as follows. As mentioned above, we shall have to carefully employ a finite IR cutoff, say, a mass parameter $\mu$ (which in our case is the external frequency). The physical IR divergences in which we are primarily interested manifest themselves in the domain $q / \mu \gg 1$ and $q / \Lambda \ll 1$, where $\mathbf{q}$ are the external momenta. By construction the latter condition is automatically satisfied in renormalized field theory. The former can also be read as $q \rightarrow \infty$ for fixed $\mu$. Note that such a scaling argument is possible only when the loop integrals are arranged in such a way that they are insensitive to their upper limits (this is what one does in renormalizing the theory). More specifically (and technically), a change in the normalization point $\mu \rightarrow b \mu$ of the theory may be interpreted as a scale transformation $q \rightarrow q / b$, and solving the Callan-Symanzik equation provides the corresponding RG flow equations. Again the fixed points with respect to these (infinitesimal) scale transformations are investigated, and the fixed point values of Wilson's functions, the so-called "anomalous dimension," yield the critical exponents.

Let us explain this for the case of the Burgers-KPZ equation. In order to renormalize the theory (i.e., remove the UV divergences) one introduces renormalized parameters, which are related to the "bare" parameters through renormalization factors containing all the UV poles. Before turning to the one-loop results, some general features of the renormalization, which are valid to every order in perturbation theory, should be noted. As discussed in Sec. II, the Ward-Takahashi identities Eqs. (2.23)-(2.26) together with Eq. (2.27) ensure that the renormalization involves only two independent renormalization factors, namely, the renormalization of the noise amplitude $D_{0}$ and the surface tension $\nu_{0}$. Hence we define renormalized parameters by

$$
D=Z_{D} D_{0}, \quad \nu=Z_{\nu} \nu_{0}
$$

and determine these by the following normalization conditions for the singular parts of the two-point vertex functions

$$
\begin{gathered}
\left.\Gamma_{\tilde{h} \tilde{h}}(\mathbf{q}, \omega)\right|_{\mathrm{NP}} ^{\text {sing }}=-2 D, \\
\left.\frac{\partial}{\partial q^{2}} \Gamma_{\tilde{h} h}(\mathbf{q}, \omega)\right|_{\mathrm{NP}} ^{\text {sing }}=\nu .
\end{gathered}
$$

The NP is conveniently chosen at $\mathbf{q}=\mathbf{0}$ and $i \omega / 2 \nu=\mu^{2}$, and $1 / \mu$ is an arbitrary length scale. We remark that this is not a minimal-subtraction prescription, as was applied by Sun and Plischke [27], where just the residues of the $1 /\left(d_{c}-d\right)$ poles (in the dimensional regularization scheme) would be included in the $Z$ factors. Rather we retain the complete dependence on the dimension $d$ in the geometric prefactors originating in the angular integrations [see the discussion following Eqs. (3.6) and (3.7)].

The corresponding Wilson functions $\zeta_{\nu}, \zeta_{D}$ and the $\beta$ function $\beta_{g}$ (see below) for the effective coupling constant

$$
g_{0}=\frac{\lambda_{0}^{2} D_{0}}{4 \nu_{0}^{3}}
$$

permit us to study the renormalization-group flows of the renormalized parameters and coupling constant $D$, $\nu$, and $g$. Note again that due to the Ward-Takahashi identities there is no renormalization for the amplitude $\lambda$ of the three-point vertex to all orders of $g_{0}$, i.e., $Z_{\lambda}=1$. Hence for the renormalized effective coupling constant $g$ one gets

$$
g=Z_{g} g_{0} C_{d} \mu^{d-2} \quad \text { with } \quad Z_{g}=Z_{D} Z_{\nu}^{-3}
$$

where we have absorbed the geometry factor $C_{d}=$ $K_{d} \Gamma(d / 2) \Gamma(2-d / 2)=\Gamma(2-d / 2) / 2^{d-1} \pi^{d / 2}$ in the definition of the renormalized coupling.

To one-loop order the bare vertex functions read

$$
\begin{aligned}
& \Gamma_{\tilde{h} \tilde{h}}(\mathbf{0}, \omega)^{\text {sing }} \\
& \quad=-2 D_{0}\left[1+\frac{\lambda_{0}^{2} D_{0}}{4 \nu_{0}^{3}} \operatorname{Re}\left(\int_{\mathbf{k}} \frac{1}{i \omega / 2 \nu_{0}+k^{2}}\right)\right], \\
& \left.\frac{\partial}{\partial q^{2}} \Gamma_{\tilde{h} \tilde{h}}(\mathbf{q}, \omega)^{\operatorname{sing}}\right|_{\mathbf{q}=\mathbf{0}} \\
& \quad=\nu_{0}\left[1-\frac{\lambda_{0}^{2} D_{0}}{4 \nu_{0}^{3}} \frac{d-2}{d} \operatorname{Re}\left(\int_{\mathbf{k}} \frac{1}{i \omega / 2 \nu_{0}+k^{2}}\right)\right],
\end{aligned}
$$

where the $d$-dependent prefactor in the latter equation is of exactly the same geometric origin as in Eq. (3.7) above. In $d=1$ the results for the amplitude of the noise spectrum and the surface tension again become identical as is required by the fluctuation-dissipation theorem valid in $d=1$. This important feature would have been lost in a minimal subtraction prescription [43]. Applying dimensional regularization, the above integrals yield at the normalization point $i \omega / 2 \nu=\mu^{2}$ :

$$
\int_{k} \frac{1}{\mu^{2}+k^{2}}=-\frac{C_{d} \mu^{d-2}}{d-2}
$$

The $1 /(d-2)=1 / \epsilon$ pole corresponds to a $\ln \Lambda$ in the cutoff-regularization scheme and to the momentum shell integral proportional to $l$ in Wilson's scheme. At this point we emphasize that this $1 / \epsilon$ pole has to be sub- 
tracted in order to renormalize the theory (i.e., remove the UV divergences corresponding to $\ln \Lambda$ at the critical dimension $d_{c}=2$ ). Let us now demonstrate once more why the dimensional regularization method (with minimal subtraction) is a dangerous procedure unless carried out with considerable precaution. Namely, one might argue that the $1 / \epsilon$ pole for the noise amplitude is canceled by the prefactor $d-2$ and hence does not have to be incorporated in the renormalization factors [27]. In our opinion, that would be incorrect. This procedure would definitely leave UV divergences in the theory, as becomes obvious in the cutoff regularization scheme, where $\ln \Lambda$ terms would survive. In summary, one should keep in mind that the $1 / \epsilon$ poles in the dimensional regularization scheme are essentially nothing else but a quite convenient way of keeping track of the $U V(\ln \Lambda)$ divergences at the critical dimension $d_{c}=2$ (the simplification is that the resulting integrals are much easier to carry out in the dimensional regularization method); one should thus strictly avoid mixing these "artificial" dimensional factors with others originating from purely geometrical properties of the integrals. Again, this might constitute a troublesome trap unless sufficient attention is paid to this issue. Henceforth, and in Appendix A 2, we try to emphasize this by clearly distinguishing between $d$ and $\epsilon$.

Using the definitions (3.22), the normalization conditions (3.23) and (3.24), and the one-loop results (3.27) and (3.28) for the singular parts of the two-point vertex functions, one arrives at

$$
\begin{aligned}
& Z_{D}=1-\frac{g_{0} C_{d} \mu^{\epsilon}}{\epsilon} \\
& Z_{\nu}=1+\left(\frac{d-2}{d}\right) \frac{g_{0} C_{d} \mu^{\epsilon}}{\epsilon}
\end{aligned}
$$

Upon defining Wilson's flow functions by

$$
\zeta_{D}=\left.\mu \frac{\partial}{\partial \mu}\right|_{0} \ln Z_{D}, \quad \zeta_{\nu}=\left.\mu \frac{\partial}{\partial \mu}\right|_{0} \ln Z_{\nu}
$$

and the $\beta$ function

$$
\beta_{g}=\left.\mu \frac{\partial}{\partial \mu}\right|_{0} g=g\left(d-2+\zeta_{D}-3 \zeta_{\nu}\right),
$$

we find the following one-loop results:

$$
\begin{aligned}
\zeta_{D} & =-g+O\left(g^{2}\right), \\
\zeta_{\nu} & =\frac{d-2}{d} g+O\left(g^{2}\right) \\
\beta_{g} & =g\left(d-2-\frac{2(2 d-3)}{d} g\right) .
\end{aligned}
$$

Note that these "physical" quantities do not contain the artificial $\epsilon$ factors anymore.

Running parameters and coupling "constants" are now defined by (see Sec. III B)

$$
\begin{aligned}
l \frac{d D}{d l} & =\zeta_{D}(l) D(l), \\
l \frac{d \nu}{d l} & =\zeta_{\nu}(l) \nu(l), \\
l \frac{d g}{d l} & =\beta_{g}(l) .
\end{aligned}
$$

We remark that these flow equations are related to the flow equations in Wilson's scheme by the following replacements [see Eqs. (3.13), (3.14), and (3.19)]:

$$
\begin{aligned}
\ln (1 / l) & \rightarrow l \\
D & \rightarrow D_{\mathrm{W}}=D e^{(z-2 \chi-d) l}, \\
\nu & \rightarrow \nu_{\mathrm{W}}=\nu e^{(z-2) l} .
\end{aligned}
$$

By determining the zeros of the $\beta$ function (3.33) we find the same fixed points $g_{0}^{*}$ and $g_{1}^{*}$ as in Wilson's scheme [Eq. (3.18), Fig. 2]; note, however, that we have incorporated somewhat different geometry factors in the definitions of the renormalized coupling $g(3.26)$ and $g_{\mathrm{W}}(3.17)$. As will be demonstrated in Sec. III B, the critical exponents are given by $z=2+\zeta_{\nu}^{*}$ and $\chi=1-\left(d+\zeta_{D}^{*}-\zeta_{\nu}^{*}\right) / 2$, respectively. This yields the identical results as the momentum shell procedure, demonstrating (to this order) that both schemes are equivalent methods in order to find the universal critical behavior.

A very important final remark is in place here. In either scheme, the above one-loop results were obtained by evaluating the integrals at fixed dimension. If an expansion near $d=2$ were applied, hardly any answer would have been found for $d<2$, due to the divergence of the fixed point $g_{1}^{*}$ at $d=3 / 2$. This is precisely the regime which is addressed by Sun and Plischke [27] in their recent two-loop calculation. They do indeed find another finite fixed point at $d=2$ with their approach, but it is difficult to see how this result may be consistent with the implicit assumption of the $\epsilon$ expansion that any nontrivial fixed point be of order $\epsilon$. Our own findings within the two-loop approximation, as explained below, rather seem to indicate that a "full" $2-\epsilon$ expansion cannot be performed consistently, in accord with the trend already seen on the one-loop level. The situation is entirely different for $d>2$. Here one may follow the ideas exploited in the study of the $\mathrm{O}(n)$ nonlinear $\sigma$ model [26] and perform an $\epsilon$ expansion above the critical dimension $d_{c}=2$. Naive power counting suggests that the theory should not be renormalizable for $d>d_{c}$, which would mean that the critical behavior could no longer be inferred from studying the UV limit of the theory, which would be ill defined. However, there appears a new fixed point of order $\epsilon$, which is IR unstable, i.e., UV stable, meaning that it governs the large-momentum behavior. Hence the theory is renormalizable despite the naive power-counting arguments. This is precisely what allows for a description of the critical properties of the model, for the IR-unstable fixed point physically corresponds to a second-order phase transition in the system. Indeed, if one inserts the one-loop fixed point value (3.20) into Eqs. (3.21) and (3.22) for the critical exponents at the roughening transition, one finds that $z_{c}=2+O\left(\epsilon^{3}\right)$ 
and $\chi_{c}=0+O\left(\epsilon^{3}\right)$, in accord with the scaling argument by Doty and Kosterlitz [18].

\section{B. Two-loop results}

In this section we return to the field-theoretic method, based on the dynamic functional (2.6), and present our results from the two-loop calculation. We shall primarily focus on the analysis and discussion of the results. For some details on the calculations and technicalities we refer the reader to the Appendix. There is, however, one important point that we have to mention here. Namely, to two-loop order the situation becomes additionally complicated with respect to the previous discussion, due to the fact that our massless field theory produces new IR singularities at $d=1$ and $d=3$ (compare Appendix A 2). These can only be handled by a "partial" $\epsilon$ expansion; i.e., we keep all the geometry factors, as explained at length above, and expand only in those $d$ dependent factors stemming from the integrals as calculated in the dimensional regularization scheme, indicated with $\epsilon$ instead of $d$ in Appendix A 2. This is, we admit, a most subtle procedure, and together with the already mentioned features, it marks the essential difference to the approach by Sun and Plischke [27]. In our opinion, however, this is the only possible method in order to approach the regime $d<2$ consistently, keeping the exactly known properties at $d=1$. For $d>2$, we shall eventually perform a "full" $\epsilon$ expansion in the final results, where all the problems cited in the preceding subsection have already been accounted for.

From the two-loop expressions for the singular parts of the two-point vertex functions (A23) and (A24) in Appendix $\mathrm{A} 1$, the normalization conditions (3.22)-(3.24), and the integrals in Appendix A 2, we find the following results for the renormalization constants:

$$
\begin{aligned}
Z_{D}=1 & -\frac{\hat{g}_{0}}{\epsilon}-(d-1) \frac{\hat{g}_{0}^{2}}{\epsilon}-\frac{d-2}{d} \frac{\hat{g}_{0}^{2}}{2 \epsilon} \\
& +(d-1) \frac{\hat{g}_{0}^{2}}{\epsilon^{2}}+O\left(\hat{g}_{0}^{3}\right), \\
Z_{\nu}=1 & +\frac{d-2}{d}\left[\frac{\hat{g}_{0}}{\epsilon}+(d-1) \frac{\hat{g}_{0}^{2}}{2 \epsilon}\right. \\
& \left.+\frac{d-2}{d} \frac{\hat{g}_{0}^{2}}{2 \epsilon}-(d-1) \frac{\hat{g}_{0}^{2}}{2 \epsilon^{2}}\right] \\
& -\frac{d-1}{d} \frac{\hat{g}_{0}^{2}}{16 \epsilon} F_{\nu}(d)+O\left(\hat{g}_{0}^{3}\right),
\end{aligned}
$$

where $\hat{g}_{0}=g_{0} C_{d} \mu^{\epsilon}$ and we have defined

$$
\begin{aligned}
F_{\nu}(d)= & 4-2(6-d) I_{00}(2)+2 d I_{10}(2) \\
& +21 I_{11}(2)-I_{21}(2)-7 I_{22}(2)+2 I_{32}(2) \\
& +4 \tilde{I}_{01}(2)-4 \tilde{I}_{12}(2)-\frac{12}{5 \sqrt{5}} \ln \frac{\sqrt{5}+1}{\sqrt{5}-1}+\frac{4}{5}
\end{aligned}
$$

The parameter integrals $I_{r s}(d)$ and $\tilde{I}_{r s}(d)$ are defined in
Appendix A 2. The corresponding Wilson flow functions Eqs. (3.32) are given by

$$
\begin{gathered}
\zeta_{D}=-g-\frac{(d-1)(2 d-1)}{d} g^{2}+O\left(g^{3}\right) \\
\zeta_{\nu}=\frac{d-2}{d} g+\frac{(d-1)(d-2)}{d} g^{2} \\
\quad-\frac{d-1}{8 d} g^{2} F_{\nu}(d)+O\left(g^{3}\right) \\
\beta_{g}=g\left(d-2-\frac{2(2 d-3)}{d} g-\frac{(d-1)(5 d-7)}{d} g^{2}\right. \\
\left.\quad-\frac{d-1}{8 d} g^{2} F_{\nu}(d)+O\left(g^{3}\right)\right)
\end{gathered}
$$

(Note that there are no $\epsilon$-dependent terms left in these expressions; specifically, there are no $1 / \epsilon$ poles, which constitutes a very nontrivial check to the calculations, along with the fact that at $d=1$ the FDT is fulfilled, as required; see Appendix A1.)

Most interestingly, the two-loop contributions to these $\zeta$ functions vanish at $d=1$. That is, there are no singular contributions to the two-point vertex functions in one dimension. This is clearly a very valuable fact for the purpose of justifying a self-consistent approximation as the mode-coupling approach, where vertex corrections are neglected [34]. In terms of the fixed points, this means that at $d=1$ the strong-coupling fixed point in the twoloop approximation is unaltered with respect to the oneloop result: $g_{1}^{*}=1 / 2$ and, of course, the critical exponents are not modified either, $z=3 / 2$ and $\chi=1 / 2$. This is reassuring, for these values already follow from a combination of the FDT (thus $\zeta_{\nu}^{*}=\zeta_{D}^{*}$ ) and the exponent identity (1.6); see Eqs. (3.57) and (3.58) below.

The flow functions (3.46) and (3.47) permit us to study the renormalization-group flow for the renormalized effective coupling constant $g$, Eq. (3.33), as a function of the dimensionality of the growth problem. The general features of this flow and the behavior of the ensuing three fixed points $g_{0}^{*}, g_{1}^{*}$, and $g_{2}^{*}$ as functions of the dimension $d$ are (Fig. 3) as follows.

(i) Just below the borderline dimension $d_{c}=2$ there are two (non-negative) fixed points, the Gaussian fixed point $g_{0}^{*}=0$, which is unstable, and one strong coupling fixed point $g_{1}^{*}$. The "weak-coupling" fixed point $g_{0}^{*}=0$, describing a smooth interface, is IR unstable. Hence, for $d<2$ the RG flow always tends to the strong-coupling point $g_{0}^{*}=0$, which is unstable, and one strong coupling fixed point $g_{1}^{*}$. The "weak-coupling" fixed point $g_{0}^{*}=0$, describing a smooth interface, is IR unstable. Hence, for $d<2$ the RG flow always tends to the strong-coupling fixed point $g_{1}^{*}$, describing a rough surface. (Below $d=1$ there is an additional fixed point in the physical region, whose value diverges for $d \rightarrow 1$. This would constitute an unstable fixed point in the flow of the coupling; it appears, however, rather doubtful to extrapolate the results of our two-loop calculations beyond $d=1$, as we had to apply the above-mentioned partial $\epsilon$ expansion.)

(ii) At the critical dimension $d_{c}=2$ the strongcoupling fixed point $g_{1}^{*}$, as obtained from the two-loop calculation, tends to infinity. It is not clear whether this 


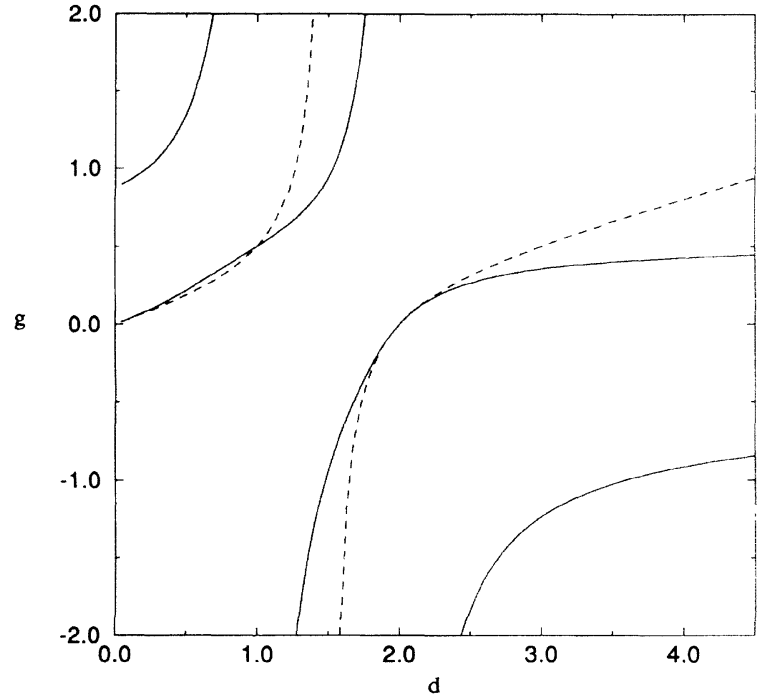

FIG. 3. Two-loop fixed points of the Burgers-KPZ equation as functions of $d$ (full lines). It is not clear if the divergences at $d=1$ and $d=2$ are inherent to the low order of perturbation expansion used, as was the case for the divergence of the one-loop fixed point (dashed here) at $d=3 / 2$, or rather if they represent a generic feature of the model.

divergence of the strong-coupling fixed point at the critical dimension $d_{c}=2$ is just an artifact of the two-loop calculation or a general feature of any finite order in perturbation theory. We suppose that it indicates that no finite strong-coupling fixed point will emerge to any order in the perturbation expansion. In this sense, there is nonperturbative strong-coupling behavior for all $d \geq 2$. There is still a considerable amount of controversy about the existence of an upper critical dimension (not to be confused with the critical dimension $d_{c}=2$ obtained from power counting arguments), at which mean field values are recovered. Actually, in view of the analogy to the nonlinear $\sigma$ model, the critical dimension $d_{c}=2$ can be regarded as the lower critical dimension, since for $d_{c}<2$ no dynamic roughening transition takes place [see (i)]. The random directed path on a Cayley tree [44] represents a possible candidate for a high-dimensional limit. The existence of a finite upper critical dimension is supported by an expansion by Cook and Derrida [45], who insert finite sections of the high-dimensional lattices in place of the nodes of the Cayley tree. The validity of such an approach has been criticized by Fisher and Huse [12], however. Also, numerical simulations [14-16] suggest that there is no upper critical dimension. From our two-loop results it is not possible to draw any decisive conclusion about the existence of an upper critical dimension. Even higher loop orders are most likely not to be very useful for deciding upon the question of the existence of an upper critical dimension. What is really needed is a systematic expansion in $1 / d$ or some other controlled expansion capable of taking into account an infinite set of diagrams. A very recent mode-coupling analysis [38], which makes no assumptions about the line shape, but is still based on an uncontrolled approximation, indeed yields $z<2$ for all $d$. (iii) Above $d_{c}=2$ our two-loop calculations support the existence of three fixed points. There are two IR stable fixed points whose domain of attraction is separated by a critical fixed point $g_{2}^{*}=g_{\mathrm{c}}^{*}$, which is IR unstable (hence UV stable). The critical fixed point $g_{c}^{*}$ describes a dynamic phase transitions and is accessible by perturbation theory (it is of order $\epsilon=d-2$ ). For $g<g_{c}^{*}$ the RG flow tends towards the weak-coupling fixed point $g_{\mathrm{WC}}^{*}=g_{0}^{*}=0$, describing a smooth interface. For effective coupling constants larger than $g_{c}^{*}$ the flow leads to an IR-stable strong-coupling fixed point $g_{2}^{*}=g_{\mathrm{SC}}^{*}$. This strong-coupling fixed point seems to be inaccessible by a perturbational approach. To two-loop order one thus finds $g_{\mathrm{SC}}^{*}=\infty$.

This scenario is similar to the results obtained for the $\mathrm{O}(n)$ nonlinear $\sigma$ model. In a $2+\epsilon$ expansion [26] one finds a nontrivial zero of the corresponding $\beta$ function. This IR-unstable fixed point defines the critical temperature in exactly the same way as the above IR-unstable fixed point in the roughening problem defines the critical coupling at which a dynamic phase transition from a smooth to a rough surface takes place. This analogy becomes even more apparent if one considers the mapping of the Burgers-KPZ equation onto the statistical mechanics of directed polymers in random media. For $d>2$ Imbrie and Spencer [46] have shown rigorously that the polymer undergoes a continuous transition from a low temperature pinned phase to a high temperature phase where the disorder is irrelevant. The above found critical fixed point controls this transition.

We now proceed to a discussion of the behavior near the different fixed points for $d>2$. In order to relate the values of the $\zeta$ functions at the fixed point to the dynamic exponent $z$ and the roughness exponent $\chi$, it is convenient to consider the two-point correlation function $C_{h h}(\mathbf{q}, \omega)$, which acquires the scaling form

$$
C_{h h}(\mathbf{q}, \omega)=q^{-d-2 \chi-z} \hat{C}\left(\omega / q^{z}\right)
$$

or equivalently (see Sec. I)

$$
C_{h h}(\mathbf{x}, t)=x^{2 \chi} \hat{C}\left(t / x^{z}\right)
$$

We want to analyze how this scaling form and the exponents for the correlation function are related to the results obtained from renormalized field theory. Since the bare vertex functions are independent of the arbitrary momentum scale $\mu$ introduced in the RG procedure, one finds the following RG equation for the two-point vertex functions $\Gamma(\mu, \nu, D, g, \mathbf{q}, \omega)$ :

$$
\left[\mu \frac{\partial}{\partial \mu}+\beta_{g} \frac{\partial}{\partial g}+\zeta_{\nu} \nu \frac{\partial}{\partial \nu}+\zeta_{D} D \frac{\partial}{\partial D}\right] \Gamma(\cdot)=0
$$

where we have introduced the abbreviation $(\cdot)=$ $(\mu, \nu, D, g, \mathbf{q}, \omega)$. The $\mathrm{RG}$ equation is readily solved with the method of characteristics. The characteristics $a(l)$ of Eq. (3.51) define running coupling "constants" and parameters into which these transform when $\mu \rightarrow \mu(l)=\mu l$. They are given by the solutions to the flow equation of the coupling $l d g / d l=\beta_{g}(l)$ and the first-order differential equations for the parameters $a=D, \nu$ 


$$
l \frac{d a(l)}{d l}=\zeta_{a}(l) a(l)
$$

with the initial conditions $D(l=1)=D$ and $\nu(l=1)=$ $\nu$, namely,

$$
a(l)=a \exp \left[\int_{1}^{l} \frac{d \rho}{\rho} \zeta_{a}(\rho)\right] .
$$

Applying a dimensional analysis one finds that $\Gamma_{\tilde{h} \tilde{h}}$ and $\Gamma_{\tilde{h} h}$ have dimensions $D$ and $\nu \mu^{2}$, respectively. Hence the solutions of the RG equations read

$$
\begin{aligned}
& \Gamma_{\tilde{h} h}(\cdot)=\mu^{2} l^{2} \nu(l) \Gamma_{\tilde{h} h}\left(\frac{\mathbf{q}}{\mu l}, \frac{\omega}{\mu^{2} l^{2} \nu(l)}, g(l)\right), \\
& \Gamma_{\tilde{h} \tilde{h}}(\cdot)=D(l) \Gamma_{\tilde{h} \tilde{h}}\left(\frac{\mathbf{q}}{\mu l}, \frac{\omega}{\mu^{2} l^{2} \nu(l)}, g(l)\right) .
\end{aligned}
$$

Since the two-point correlation function is related to the two-point vertex function by $C=\Gamma_{\tilde{h} \tilde{h}} /\left|\Gamma_{\tilde{h} h}\right|^{2}$, one gets at a fixed point, with the matching condition $\mathbf{q} / \mu l=1$,

$$
C(\mu, \nu, D, g, \mathbf{q}, \omega)=q^{-4-2 \zeta_{\nu}^{*}+\zeta_{D}^{*}} \hat{C}\left(\frac{\omega}{q^{2+\zeta_{\nu}^{*}}}\right)
$$

Hence we arrive at the following already mentioned relations:

$$
\begin{aligned}
& \chi=1-\frac{d}{2}+\frac{\zeta_{\nu}^{*}-\zeta_{D}^{*}}{2} \\
& z=2+\zeta_{\nu}^{*}
\end{aligned}
$$

At the weak-coupling fixed point $g_{0}^{*}=0$, we have $\chi=$ $1-d / 2$ and $z=2$. At any nonzero fixed point $g^{*} \neq 0$ one gets

$$
d-2+\zeta_{D}^{*}-3 \zeta_{\nu}^{*}=0
$$

from $\beta_{g}\left(g^{*}\right)=0$. Note that this exponent identity results ultimately from the Galilean invariance of the BurgersKPZ equation. Hence we find

$$
\begin{aligned}
& \chi=-\zeta_{\nu}^{*} \\
& z=2+\zeta_{\nu}^{*}
\end{aligned}
$$

From these relations one can easily infer the exponent identity $\chi+z=2$ already mentioned in Sec. I.

Let us now investigate the two-loop results at the IRunstable (critical) fixed point $g_{\mathrm{c}}^{*}$. One can show that $F_{\nu}(d)=8+O(\epsilon)$, where the $O(\epsilon)$ coefficient can also be determined numerically $F_{\nu}(d) \approx 8-4.0797 \epsilon$ [see Eq. (A38)]. Hence one finds that in a consistent expansion in both $g$ and $\epsilon$

$$
\begin{aligned}
\zeta_{D} & =-g-\frac{3}{2} g^{2}+O\left(g^{3}, g^{2} \epsilon, g \epsilon^{2}, \epsilon^{3}\right) \\
\zeta_{\nu} & =\frac{\epsilon}{2} g-\frac{1}{2} g^{2}+O\left(g^{3}, g^{2} \epsilon, g \epsilon^{2}, \epsilon^{3}\right),
\end{aligned}
$$

where $\epsilon=d-2$. With Eq. (3.33) this yields, for the $\beta$ function,

$$
\beta_{g}=g\left(\epsilon-\frac{2+3 \epsilon}{2} g+O\left(g^{3}, g^{2} \epsilon, g \epsilon^{2}, \epsilon^{3}\right)\right)
$$

which is identical to the one-loop function since all the $O\left(g^{2}\right)$ corrections cancel. The resulting critical fixed point is $g_{c}^{*}=\epsilon-3 \epsilon^{2} / 2+O\left(\epsilon^{3}\right)$ and there is no finite strong-coupling fixed point. Inserting this result for the unstable fixed point into the $\zeta$ functions one realizes that all $O\left(\epsilon^{2}\right)$ corrections cancel, i.e.,

$$
\begin{gathered}
\zeta_{D}^{*}=-\epsilon+O\left(\epsilon^{3}\right) \\
\zeta_{\nu}^{*}=0+O\left(\epsilon^{3}\right) .
\end{gathered}
$$

Hence our two-loop calculations confirm the results by Doty and Kosterlitz [18] that $z_{c}=2$ and $\chi_{c}=0$ at the roughening transition. Note that in our perturbation expansion this result is due to a most remarkable and not at all obvious cancellation of very different contributions. This is a very reassuring feature of our method again, at least if one follows the considerations in Ref. [18].

Now let us reverse the argument and assume that $z_{c}=$ 2 is an exact result at the critical fixed point. Then, with Eqs. (3.59) and (3.61) one gets at the weak-coupling fixed point

$$
\begin{aligned}
(d-2)+\zeta_{D}\left(g_{\mathrm{c}}^{*}\right) & =0, \\
\zeta_{\nu}\left(g_{\mathrm{c}}^{*}\right) & =0 .
\end{aligned}
$$

Therefore, it should also be possible to determine the value of the critical fixed point from one of the latter equations. In fact, one obtains from Eq. (3.67) the same critical fixed point as from the zero of the $\beta$ function. However, in order to determine the value of $g_{c}^{*}$ up to terms of order $O\left(\epsilon^{3}\right)$ from Eq. (3.68) one needs the coefficient of the $O\left(g^{2}\right)$ term and the $O\left(g^{3}\right)$ term of $\zeta_{\nu}$ up to terms of order $O\left(\epsilon^{2}\right)$ and $O\left(\epsilon^{1}\right)$, respectively. For the calculation of the fixed point value from the zeros of the $\beta$ function, we had to know the $O\left(g^{2}\right)$ term of $\zeta_{\nu}$ up to terms of order $O(\epsilon)$, only. From Eq. (3.47) one obtains upon including an unknown three-loop term

$$
\zeta_{\nu}=\frac{g}{d}\left[\epsilon+\left(-1+\frac{4.0797}{8} \epsilon\right) g+A g^{2}\right]
$$

where $A$ is a constant of order $O(1)$. Equation (3.68) yields, for the critical fixed point, $g_{c}^{*}=\epsilon-3 \epsilon^{2} / 2$, only if $A=-(3 / 2+4.0797 / 8)$, i.e., one can determine the three-loop correction for the renormalization of the surface tension $\nu$.

Let us comment on the $d$ dependence of the critical fixed point. Keeping the full dependence of the $\zeta$ function for the noise amplitude on $d$, one obtains, from Eqs. (3.46) and (3.67), for the critical fixed point

$$
g_{c}^{*}=\frac{-1+\sqrt{1+4(d-1)(2 d-1)(d-2) / d}}{2(d-1)(2 d-1) / d}
$$

which in the limit of large $d$ reduces to a finite value $g_{c}^{*} \rightarrow 1 / \sqrt{2}$. It would be interesting to see how this $d$ dependence of the fixed point compares with numerical simulations. Note, however, that the scale of $g_{c}^{*}$ is nonuniversal and depends on the precise definition of the coupling $g$.

We close this section by a discussion of the RG equa- 
tions for $d>2$ and its implications on the kinetic roughening transition described by the IR-unstable (repulsive) fixed point $g_{c}^{*}$. We remark that a detailed analysis of the crossover scaling behavior in the weak-coupling regime at the one-loop level has been given by Nattermann and Tang $[20]$. We have shown above that the exponents at the transition are given by $z_{c}=2+O\left(\epsilon^{3}\right)$ and $\chi_{c}=0+O\left(\epsilon^{3}\right)$. In the smooth phase (i.e., for coupling constants $g<g_{c}^{*}$ ) the effective coupling approaches zero. Therefore, the long-distance and long-time properties in this phase may be obtained from a perturbative RG study. (In the rough phase a perturbative expansion is not possible due to the strong-coupling behavior.)

The proximity to the critical point can be measured in terms of the control parameter $\delta=\left(g_{c}^{*}-g\right) / g_{c}^{*}$, and, quite analogously to the treatment of the nonlinear $\sigma$ model at its critical point [26], we define a correlation length $\xi(g)$ via the solution of the differential equation

$$
\beta_{g}(g) \frac{d \xi(g)}{d g}=\xi(g)
$$

Since $\xi$ has the dimension of a length this can also be written as

$$
\left[\mu \frac{\partial}{\partial \mu}+\beta_{g}(g) \frac{\partial}{\partial g}\right] \xi(g, \mu)=0
$$

i.e.,

$$
\begin{aligned}
\xi(g, \mu)= & \mu^{-1} g^{1 /(d-2)} \\
& \times \exp \left[\int_{0}^{g} d g^{\prime}\left(\frac{1}{\beta_{g}\left(g^{\prime}\right)}-\frac{1}{(d-2) g^{\prime}}\right)\right] .
\end{aligned}
$$

Close to the critical fixed point we can write

$$
\frac{d \xi}{d g}=\frac{\xi}{\beta_{g}(g(l))}=\frac{\xi}{\left[g(l)-g_{c}^{*}\right] \beta_{g}^{\prime}\left(g_{c}^{*}\right)},
$$

the solution of which is given by

$$
\xi(g) \propto\left|g-g_{c}^{*}\right|^{1 / \beta_{g}^{\prime}\left(g_{c}^{*}\right)} \propto|\delta|^{-\nu}
$$

where $\beta_{g}^{\prime}\left(g_{c}^{*}\right)=d \beta_{g}(g) /\left.d g\right|_{g_{c}^{*}}$. Hence the critical exponent for the correlation length is determined by the first derivative of the $\beta$ function at the critical fixed point $\nu=-1 / \beta_{g}^{\prime}\left(g_{c}^{*}\right)$. Since we have shown above that all twoloop contributions to this $\beta$ function cancel, we find that the correlation length exponent is

$$
1 / \nu=\epsilon+O\left(\epsilon^{3}\right)
$$

To one-loop order, this result was already obtained by Nattermann and Tang [20], who also discuss how the above scaling is modified to a logarithmic form at $d_{c}=2$.

Let us now solve the RG equations, Eq. (3.51), in such a way that the scaling behavior at the dynamic phase transition becomes apparent (see also Ref. [20]). Dimensional analysis tells us that

$$
\begin{aligned}
& \Gamma_{\tilde{h} \tilde{h}}(\mu, \nu, D, g, \mathbf{q}, \omega)=D \hat{\Gamma}_{\tilde{h} \tilde{h}}\left(\frac{q}{\mu}, \frac{\omega}{\mu^{2} \nu}\right) \\
& \Gamma_{\tilde{h} h}(\mu, \nu, D, g, \mathbf{q}, \omega)=\mu^{2} \nu \hat{\Gamma}_{\tilde{h} h}\left(\frac{q}{\mu}, \frac{\omega}{\mu^{2} \nu}\right)
\end{aligned}
$$

Thus the solution of the RG equation can be written as

$$
\begin{aligned}
& \Gamma_{\tilde{h} \tilde{h}}(\cdot)=D(g) \gamma_{\tilde{h} \tilde{h}}\left(q \xi(g), \frac{\omega \xi^{2}(g)}{\nu(g)}\right) \\
& \Gamma_{\tilde{h} h}(\cdot)=\xi(g)^{-2} \nu(g) \gamma_{\tilde{h} h}\left(q \xi(g), \frac{\omega \xi^{2}(g)}{\nu(g)}\right),
\end{aligned}
$$

with

$$
\begin{aligned}
& D(g)=D \exp \left\{-\int_{0}^{g} \frac{d g^{\prime}}{\beta\left(g^{\prime}\right)} \zeta_{D}\left(g^{\prime}\right)\right\}, \\
& \nu(g)=\nu \exp \left\{-\int_{0}^{g} \frac{d g^{\prime}}{\beta\left(g^{\prime}\right)} \zeta_{\nu}\left(g^{\prime}\right)\right\} .
\end{aligned}
$$

In summary, one obtains the following dynamic scaling behavior of the correlation function:

$$
C(\delta, \mathbf{q}, \omega)=M(\xi(g)) \xi^{4}(g) \hat{C}\left(q \xi(g), \frac{\omega}{\omega_{c}(\xi(g))}\right)
$$

where we have defined the quantity

$$
M(\xi(g))=\exp \left\{-\int_{0}^{g} \frac{d g}{\beta(g)}\left[\zeta_{D}(g)-2 \zeta_{\nu}(g)\right]\right\} .
$$

We have also introduced a characteristic frequency by

$$
\omega_{c}(\xi(g))=\mu^{2} \xi^{-2} \exp \left\{-\int_{0}^{g} \frac{d g}{\beta(g)} \zeta_{\nu}(g)\right\} .
$$

Close to the critical fixed point the latter equation reduces to $\omega_{c}(\xi(g)) \propto \xi^{-z_{c}}$, with the dynamical critical exponent $z_{c}=2+\zeta_{\nu}^{*}$. One should note that this dynamic scaling form is quite analogous to the corresponding scaling in the nonlinear $\sigma$ model [26] and the same conclusions drawn there apply for the roughening transition as well. Let us contrast the above scaling behavior with the well-known scaling behavior of a standard $\phi^{4}$ model close to its critical point. The $\phi^{4}$ model depends on two coupling constants: the coefficient of the $\phi^{2}$ term, which plays the role of the control parameter (reduced temperature) corresponding to the reduced effective coupling $\delta$ defined above, and the coefficient of the $\phi^{4}$ term, which has no equivalent here. The scaling form of the 
$\phi^{4}$ model is obtained at the $I R$-stable fixed point, e.g., obtained by an $\epsilon$ expansion near the upper critical dimension 4. In the present case, however, there is not only scaling behavior at the critical fixed point, but in the entire "smooth" phase. This behavior, Eq. (3.83), is most similar to that of $\mathrm{O}(n)$ symmetric models in the low temperature phase and more closely resembles the form known from crossover scaling behavior [20]. In order to study the crossover scaling behavior in the weak-coupling phase, we use the following "resummed" expressions for the $\zeta$ and $\beta$ functions:

$$
\begin{aligned}
\zeta_{D} & =-\frac{g}{1-3 / 2 g}, \\
\zeta_{\nu} & =\frac{g}{d}\left(\epsilon-\frac{g}{1-3 / 2 g}\right), \\
\beta_{g} & =g\left(\epsilon-\frac{2+3 \epsilon}{2} g\right),
\end{aligned}
$$

which are identical to the expressions above, but also take into account that the fixed point values can be equally well obtained from the zero of the $\beta$ function and Eqs. (3.67) and (3.68).

First, we consider the crossover of the correlation length as a function of the effective coupling $g$. As depicted in Fig. 4, the correlation length crosses over from $\xi \propto g^{1 / \epsilon}$ at small couplings to $\xi \propto\left|g-g_{c}^{*}\right|^{-\nu}$ as $g$ approaches the critical coupling $g_{c}^{*}$. The crossover function shown in Fig. 4 is universal, i.e., all the nonuniversal scales may be absorbed in an amplitude $\xi_{0}$ for the correlation length. Specifically, the location of the crossover $g_{\text {cross }}$ can be obtained from Fig. 4 . Similar crossover behavior is found for the noise amplitude $D$ and the surface tension $\nu$, as shown in Fig. 5. The asymptotic behavior at the critical fixed point is given by

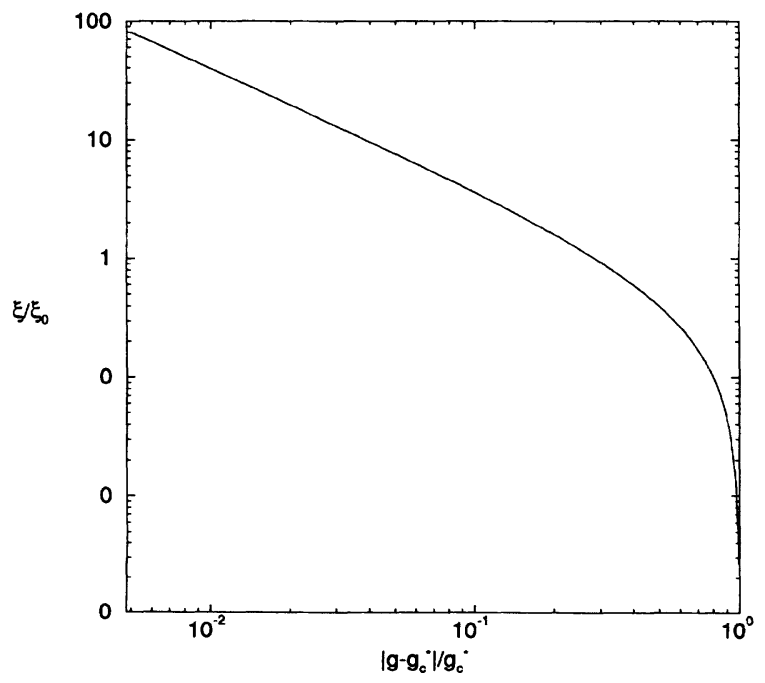

FIG. 4. The correlation length $\xi$ in the smooth phase versus $\left|g-g_{c}^{*}\right| / g_{c}^{*}$. The correlation length crosses over from $\xi \propto g^{1 / \epsilon}$ at small couplings to $\xi \propto\left|g-g_{c}^{*}\right|^{-\nu}$ as $g$ approaches the critical coupling $g_{c}^{*}$. In the figure we have set $\epsilon=1$.

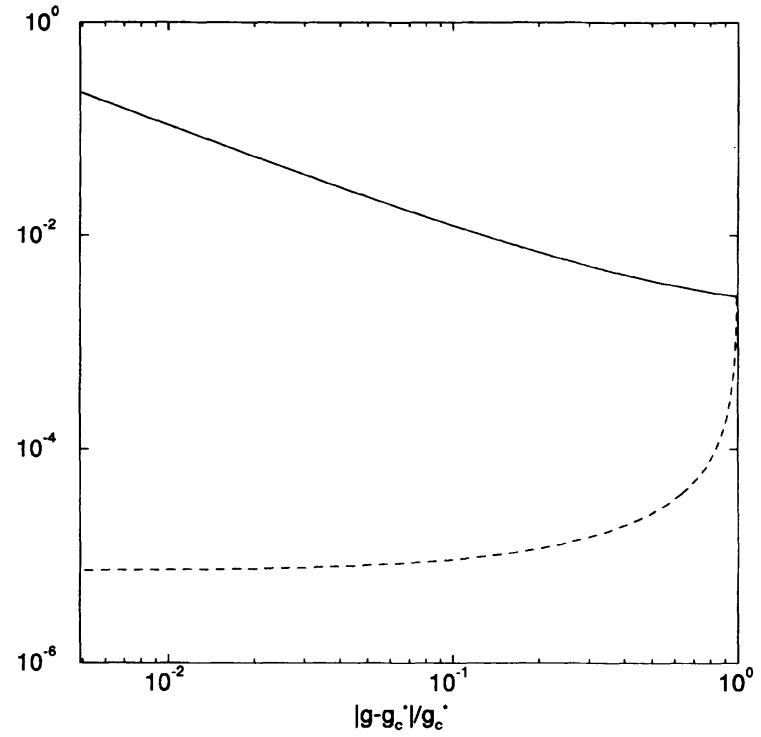

FIG. 5. The renormalized noise amplitude $D(g)$ (solid line) and surface tension $\nu(g)$ (dashed line) (up to a nonuniversal amplitude) in the smooth phase versus $\left|g-g_{c}^{*}\right| / g_{c}^{*}$. The noise amplitude shows a crossover from an exponential increase $D(g) \propto e^{g / \epsilon}$ at small values of $g$ to a power law divergence $D(g) \propto\left|g-g_{c}^{*}\right|^{-\epsilon \nu}$ as $g$ approaches the critical coupling $g_{c}^{*}$. The renormalized surface tension $\nu(g)$ crosses over from exponentially decreasing $\nu(g) \propto e^{-g / 2}$ to a constant at criticality. The curves are plotted for $d=3$ (i.e., $\epsilon=1$ ).

$$
\begin{aligned}
D(g) & \propto\left|g-g_{c}^{*}\right|^{-\epsilon \nu}=\left|g-g_{c}^{*}\right|^{-1}, \\
\nu(g) & \propto\left|g-g_{c}^{*}\right|^{0} .
\end{aligned}
$$

The noise amplitude shows a crossover from an exponential increase $D(g) \propto e^{g / \epsilon}$ at small values of $g$ to a power law divergence $D(g) \propto\left|g-g_{c}^{*}\right|^{-\epsilon \nu}$ as $g$ approaches the critical coupling $g_{c}^{*}$. The renormalized sur-

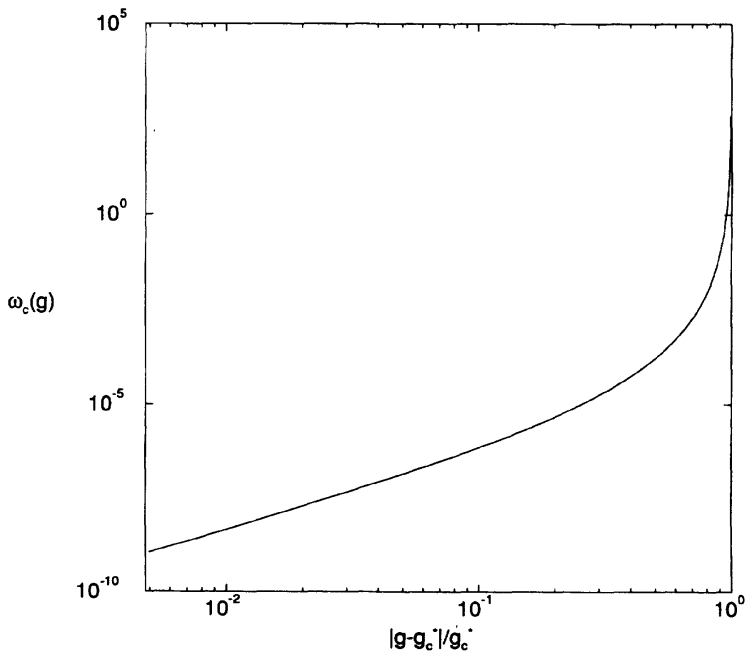

FIG. 6. The characteristic frequency $\omega_{c}(g)$ (up to a nonuniversal amplitude) in the smooth phase versus $\left|g-g_{c}^{*}\right| / g_{c}^{*}$. The characteristic frequency crosses over from $\omega_{c}(g) \propto g^{-2 / \epsilon} e^{-g / 2}$ at small couplings to $\omega_{c}(g) \propto\left|g-g_{c}^{*}\right|^{-2}$ as $g$ approaches the critical coupling $g_{c}^{*}$. The figure shows the result for $\epsilon=1$. 
face tension $\nu(g)$ crosses over from exponentially decreasing $\nu(g) \propto e^{-g / 2}$ to a constant at criticality. Finally, the crossover behavior of the characteristic frequency is depicted in Fig. 6. It crosses over from $\omega_{c}(g) \propto g^{-2 / \epsilon} e^{-g / 2}$ at small couplings to $\omega_{c}(g) \propto\left|g-g_{c}^{*}\right|^{-2}$ as $g$ approaches the critical coupling $g_{c}^{*}$.

It would be interesting to test the validity of the above crossover scaling, including the form of the crossover functions, by numerical simulation.

\section{SUMMARY AND CONCLUSIONS}

In this paper we have given a systematic analysis of the Burgers-KPZ equation in $d+1$ dimensions by dynamic renormalization-group theory. We have paid special attention to the interconnections of the various renormalization group techniques.

Let us briefly summarize our main conclusions. For the roughening dynamics as described by the Burgers-KPZ equation we find the following results: (1) The roughening transition of the Burgers-KPZ equation is understood in terms of an IR-unstable fixed point above the (lower) critical dimension $d_{c}=2$. The value of this critical fixed point is accessible by a $2+\epsilon$ expansion, similar to an analogous expansion for the nonlinear $\sigma$ model. The critical properties of this transition are characterized by one independent exponent. Doty and Kosterlitz have argued on the basis of a standard scaling argument that the dynamic exponent $z_{c}$ at the dynamic roughening transition should be exactly equal to 2 . From our two-loop calculation we find that $z_{c}=2+O\left(\epsilon^{3}\right)$, which supports their considerations. We have further analyzed the scaling behavior near the transition and in the smooth phase. Introducing a control parameter $\delta=\left(g_{c}^{*}-g\right) / g_{c}^{*}$, which measures the distance to the critical point, one can define a correlation length $\xi$ and calculate the corresponding exponent $\nu$. We find that $\nu=1 / \epsilon+O\left(\epsilon^{3}\right)$. (2) Below the borderline dimension $d_{c}=2$ there appear two fixed points, an IR-unstable Gaussian fixed point and an IRstable strong-coupling fixed point. Note that the strongcoupling fixed point is not of order $O(\epsilon)$ and there is apparently also no other known small parameter. Hence the phase described by this strong-coupling fixed point is not accessible by a controlled perturbation theory and one needs nonperturbative methods (e.g., mode-coupling theories) to access the physics of the rough phase. The weak-coupling fixed point describes a smooth interface. But, since this fixed point is always unstable, the interface is always rough below $d_{c}=2$. (3) At the critical dimension the strong-coupling fixed point, as obtained from the two-loop calculation, tends to infinity. It is not clear, whether this divergence of the strong-coupling fixed point is just an artifact of the two-loop calculation or a general feature of any finite order in perturbation theory. We suppose that it indicates that there is nonperturbative strong-coupling behavior for all $d \geq 2$.

We have also highlighted the importance of carefully considering the structure of the dimensional regularization method. As explained in detail in Sec. III, some care is required in using this method, especially in distinguish- ing between the dimensional dependence contained in $1 / \epsilon$ poles and $d$-dependent factors originating from purely geometric properties of the loop integrals. Whereas the $1 / \epsilon$ poles constitute essentially nothing but a quite convenient way of keeping track of the UV divergences in the perturbation expansion, all other $d$-dependent factors characterize the symmetry and internal structure of the model. Note that with these precautions the results for the renormalization factors quite naturally obey the FDT in $d=1$ dimension; it is not necessary to perform a separate treatment of this case.

The two-loop calculations allow for no decisive conclusion about the existence of an upper critical dimension, at which mean field values for the exponents are recovered. To decide upon the existence of an upper critical dimension it would be highly useful to do a systematic expansion in $1 / d$ within the framework of the BurgersKPZ equation. To our knowledge, such an expansion is not yet available at the present time.

Very recent results by $\mathrm{Tu}$ [38], who has solved the mode-coupling equations without assuming any specific line shape, indicate that $z<2$ for all dimensions $d$. However, the results obtained from mode-coupling theories are not quite conclusive, since it is not known in what sense such theories constitute a controlled expansion. Nevertheless, resummation techniques such as the mode-coupling theory are at present the only available analytical technique to study strong-coupling behavior.

For the $(1+1)$-dimensional case there is very close agreement between numerical simulations and the results obtained from mode-coupling theory [34]. Our two-loop calculations demonstrate that for $d=1$ all singular twoloop corrections vanish. This is a highly nontrivial result, because for this to be the case, the vertex corrections entering the calculations of the correlation and response functions must cancel each other. It is exactly those vertex corrections, which are neglected by mode-coupling theory, however. Hence our RG analysis provides a distinct hint why this approach has been so successful in $1+1$ dimension. Yet further analysis is required to clarify this point [47].

Summarizing, it would thus be of interest to further investigate the behavior of the strong-coupling fixed point by a systematic $1 / d$ expansion, as well as by modecoupling theory in high dimensions. In addition, it would be interesting to know how the value of the critical fixed point (obtained within the two-loop calculation)especially its $d$ dependence-and the crossover scaling analysis in the smooth phase compare with results from numerical simulations.

\section{ACKNOWLEDGMENTS}

It is a pleasure to acknowledge helpful discussions with T. Hwa, D.S. Fisher, M. Kardar, T. Nattermann, G. Zumbach, L. Balents, and T. Sun. The work of E.F. and U.C.T has been supported by the Deutsche Forschungsgemeinschaft under Contracts Nos. Fr. 850/2-1,2 and Ta. 177/1-1, respectively. We also ac- 


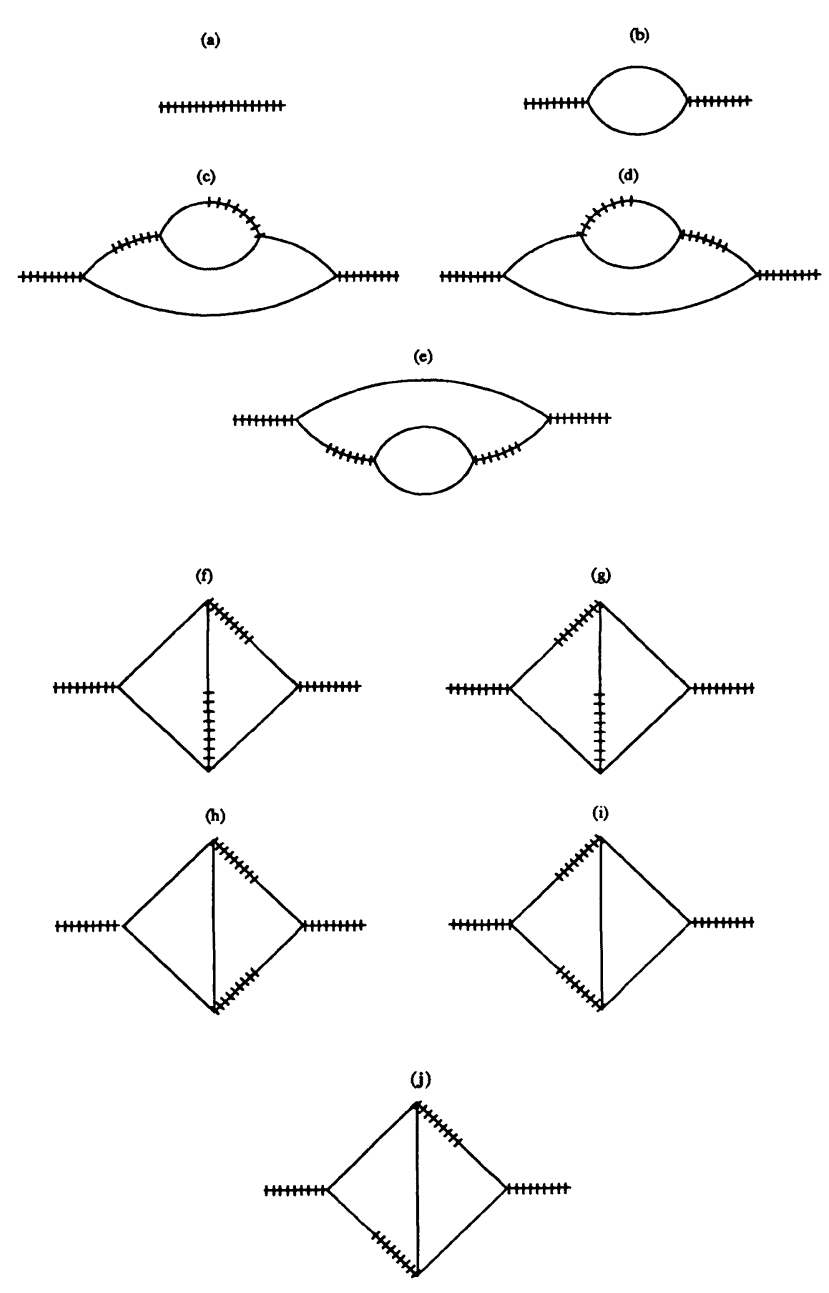

FIG. 7. Feynman diagrams for the dynamical perturbation expansion of $\Gamma_{\tilde{h} \tilde{h}}(\mathbf{q}, \omega)$ to two-loop order.

knowledge support by the NSF through Harvard University's Materials Research Laboratory and through Grant No. DMR-91-15491.

\section{APPENDIX A}

In this appendix, we list the explicit results for the perturbation theory to two-loop order. Appendix A 1 comprises the Feynman diagrams and the corresponding analytical expressions, while we present the evaluation of the relevant integrals in dimensional regularization in Appendix A 2.
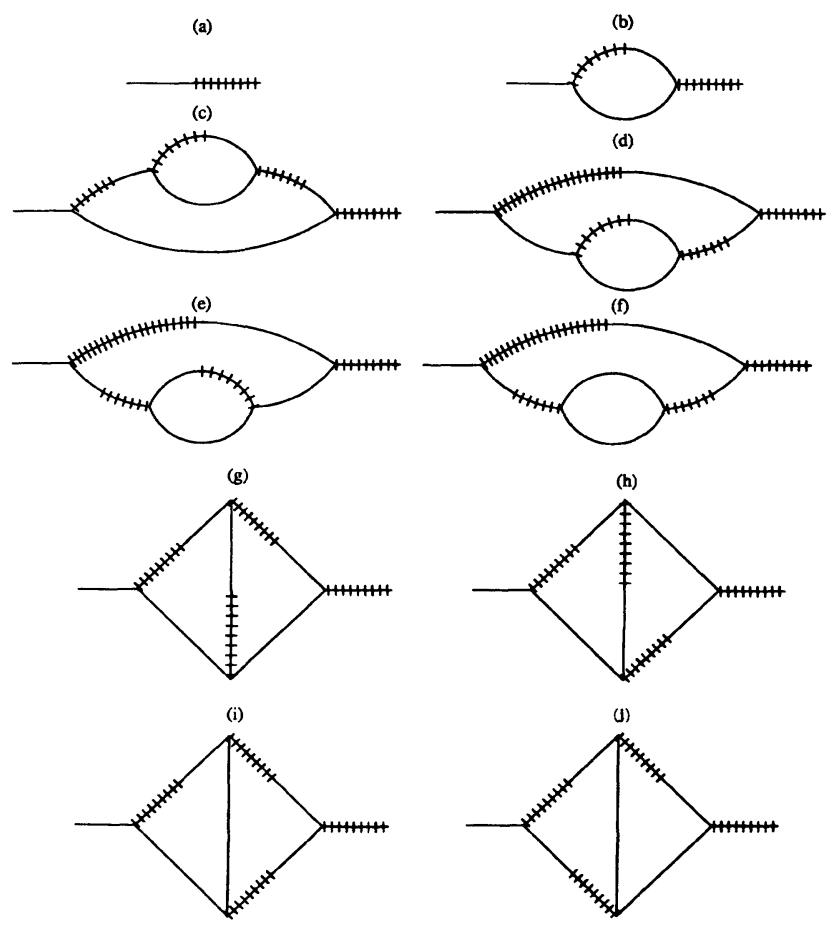

FIG. 8. Feynman diagrams for the dynamical perturbation expansion of $\Gamma_{\tilde{h} h}(\mathbf{q}, \omega)$ to two-loop order.

\section{Two-loop perturbation theory for the two-point vertex functions}

This section comprises the Feynman diagrams to twoloop order for the Burgers-Kardar-Parisi-Zhang equation and the corresponding momentum integrals. The integrations over the internal frequencies have already been performed using the residue theorem.

We start with a list of the contributions to two-loop order to the fully wave-vector- and frequency-dependent two-point vertex functions. In writing down the diagrammatic expansion for the dynamic functional one has to take into account restrictions which follow from causality. In Sec. II we have not written down the Jacobian $\mathcal{J}[h]=\mathcal{D}[\eta] / \mathcal{D}[h]$. In general the Jacobian depends on the discretization of the Langevin equation (needed to give a proper definition to the path integral). As can be shown quite generally [22] the Jacobian cancels the equal time contractions of the field $h$ and the response field $\tilde{h}$. Keeping in mind (or by choosing a discretization with the Jacobian equal to 1) one can omit the Jacobian in the dynamic functional. The Feynman diagrams, which account for the restrictions imposed by causality [48], are depicted in Figs. 7 and 8 for $\Gamma_{\tilde{h} \tilde{h}}(\mathbf{q}, \omega)$ and $\Gamma_{\tilde{h} h}(\mathbf{q}, \omega)$, respectively. In case of the former, adding the terms corresponding to both opposite time directions, respectively, provides some simplifications. The corresponding analytical expressions are, for $\Gamma_{\tilde{h} \tilde{h}}(\mathbf{q}, \omega)$ and $\Gamma_{\tilde{h} h}(\mathbf{q}, \omega)$, respectively,

$(a)+(b)=-2 D_{0}-\frac{\lambda_{0}^{2} D_{0}^{2}}{2 \nu_{0}^{3}} \operatorname{Re} \int_{p} \frac{\left(q^{2} / 4-p^{2}\right)^{2}}{(\mathbf{q} / 2+\mathbf{p})^{2}(\mathbf{q} / 2-\mathbf{p})^{2}} \frac{1}{i \omega / 2 \nu_{0}+q^{2} / 4+p^{2}}$, 
$(c)+(d)=\frac{\lambda_{0}^{4} D_{0}^{3}}{8 \nu_{0}^{6}} \operatorname{Re} \int_{p} \frac{\left(q^{2} / 4-p^{2}\right)^{2}}{(\mathbf{q} / 2+\mathbf{p})^{2}(\mathbf{q} / 2-\mathbf{p})^{4}} \int_{k} \frac{(\mathbf{q} / 4-\mathbf{p} / 2)^{2}-k^{2}}{i \omega / 2 \nu_{0}+q^{2} / 4+p^{2}}$

$\times \frac{(\mathbf{q} / 2-\mathbf{p})^{2}\left[(\mathbf{q} / 4-\mathbf{p} / 2)^{2}+k^{2}\right]-2[(\mathbf{q k}) / 2-(\mathbf{p k})]^{2}}{(\mathbf{q} / 4-\mathbf{p} / 2+\mathbf{k})^{2}(\mathbf{q} / 4-\mathbf{p} / 2-\mathbf{k})^{2}\left[3(\mathbf{q} / 2-\mathbf{p})^{2} / 4+k^{2}\right]}$

$\times\left[2+\frac{(\mathbf{q} / 2-\mathbf{p})^{2}}{i \omega / 2 \nu_{0}+(\mathbf{q} / 2+\mathbf{p})^{2} / 2+(\mathbf{q} / 2-\mathbf{p})^{2} / 4+k^{2}}\left(1+\frac{3(\mathbf{q} / 2-\mathbf{p})^{2} / 4+k^{2}}{i \omega / 2 \nu_{0}+q^{2} / 4+p^{2}}\right)\right]$,

$(e)=-\frac{\lambda_{0}^{4} D_{0}^{3}}{8 \nu_{0}^{6}} \operatorname{Re} \int_{p} \frac{\left(q^{2} / 4-p^{2}\right)^{2}}{(\mathbf{q} / 2+\mathbf{p})^{2}(\mathbf{q} / 2-\mathbf{p})^{2}} \frac{1}{i \omega / 2 \nu_{0}+q^{2} / 4+p^{2}}$

$\times \int_{k} \frac{\left[(\mathbf{q} / 4-\mathbf{p} / 2)^{2}-k^{2}\right]^{2}}{(\mathbf{q} / 4-\mathbf{p} / 2+\mathbf{k})^{2}(\mathbf{q} / 4-\mathbf{p} / 2-\mathbf{k})^{2}\left[3(\mathbf{q} / 2-\mathbf{p})^{2} / 4+k^{2}\right]}$

$\times\left(2+\frac{(\mathbf{q} / 2-\mathbf{p})^{2}}{i \omega / 2 \nu_{0}+(\mathbf{q} / 2+\mathbf{p})^{2} / 2+(\mathbf{q} / 2-\mathbf{p})^{2} / 4+k^{2}}\right)$,

$(f)+(g)=-\frac{\lambda_{0}^{4} D_{0}^{3}}{\nu_{0}^{6}} \operatorname{Re} \int_{p} \frac{q^{2} / 4-p^{2}}{(\mathbf{q} / 2+\mathbf{p})^{2}(\mathbf{q} / 2-\mathbf{p})^{2}} \int_{k} \frac{(\mathbf{q k}) / 2+(\mathbf{p k})}{(\mathbf{q} / 2-\mathbf{p}-\mathbf{k})^{2}}$

$\times \frac{q^{2} / 4-(\mathbf{p}+\mathbf{k})^{2}}{i \omega / 2 \nu_{0}+q^{2} / 4+(\mathbf{p}+\mathbf{k})^{2}} \frac{(\mathbf{q k}) / 2-(\mathbf{p k})-(\mathbf{q} / 2-\mathbf{p})^{2}}{(\mathbf{q} / 2-\mathbf{p}-\mathbf{k})^{2}+(\mathbf{q} / 2-\mathbf{p})^{2}+k^{2}}$

$\times\left(\operatorname{Re} \frac{1}{i \omega / 2 \nu_{0}+q^{2} / 4+p^{2}}+\frac{1}{i \omega / 2 \nu_{0}+q^{2} / 4+p^{2}} \frac{(\mathbf{q} / 2-\mathbf{p})^{2}}{i \omega / \nu_{0}+(\mathbf{q} / 2-\mathbf{p}-\mathbf{k})^{2}+(\mathbf{q} / 2+\mathbf{p})^{2}+k^{2}}\right)$,

$(h)+(i)=\frac{\lambda_{0}^{4} D_{0}^{3}}{\nu_{0}^{6}} \operatorname{Re} \int_{p} \frac{q^{2} / 4-p^{2}}{(\mathbf{q} / 2+\mathbf{p})^{2}(\mathbf{q} / 2-\mathbf{p})^{2}} \int_{k} \frac{(\mathbf{q k})^{2} / 4-(\mathbf{p k})^{2}}{k^{2}}$

$\times \frac{q^{2} / 4-(\mathbf{p}+\mathbf{k})^{2}}{i \omega / 2 \nu_{0}+q^{2} / 4+(\mathbf{p}+\mathbf{k})^{2}} \frac{1}{(\mathbf{q} / 2-\mathbf{p}-\mathbf{k})^{2}+(\mathbf{q} / 2-\mathbf{p})^{2}+k^{2}}$

$\times\left(\operatorname{Re} \frac{1}{i \omega / 2 \nu_{0}+q^{2} / 4+p^{2}}+\frac{1}{i \omega / 2 \nu_{0}+q^{2} / 4+p^{2}} \frac{(\mathbf{q} / 2-\mathbf{p})^{2}}{i \omega / \nu_{0}+(\mathbf{q} / 2-\mathbf{p}-\mathbf{k})^{2}+(\mathbf{q} / 2+\mathbf{p})^{2}+k^{2}}\right)$,

$(j)=-\frac{\lambda_{0}^{4} D_{0}^{3}}{2 \nu_{0}^{6}} \operatorname{Re} \int_{p} \frac{q^{2} / 4-p^{2}}{(\mathbf{q} / 2+\mathbf{p})^{2}} \int_{k} \frac{(\mathbf{q k}) / 2+(\mathbf{p k})}{k^{2}(\mathbf{q} / 2-\mathbf{p}-\mathbf{k})^{2}} \frac{q^{2} / 4-(\mathbf{p}+\mathbf{k})^{2}}{i \omega / 2 \nu_{0}+q^{2} / 4+(\mathbf{p}+\mathbf{k})^{2}} \frac{(\mathbf{q k}) / 2-(\mathbf{p k})-k^{2}}{(\mathbf{q} / 2-\mathbf{p}-\mathbf{k})^{2}+(\mathbf{q} / 2-\mathbf{p})^{2}+k^{2}}$

$\times\left(\frac{1}{-i \omega / 2 \nu_{0}+q^{2} / 4+p^{2}}+\frac{2}{i \omega / \nu_{0}+(\mathbf{q} / 2-\mathbf{p}-\mathbf{k})^{2}+(\mathbf{q} / 2+\mathbf{p})^{2}+k^{2}}\right) ;$

$(a)+(b)=i \omega+\nu_{0} q^{2}+\frac{\lambda_{0}^{2} D_{0}}{4 \nu_{0}^{2}} \int_{p} \frac{q^{2} / 4-p^{2}}{(\mathbf{q} / 2-\mathbf{p})^{2}} \frac{q^{2}-2(\mathbf{q p})}{i \omega / 2 \nu_{0}+q^{2} / 4+p^{2}}$,

$(c)=-\frac{\lambda_{0}^{4} D_{0}^{2}}{32 \nu_{0}^{5}} \int_{p} \frac{q^{2} / 4-p^{2}}{(\mathbf{q} / 2+\mathbf{p})^{2}} \frac{q^{2}+2(\mathbf{q} \mathbf{p})}{\left(i \omega / 2 \nu_{0}+q^{2} / 4+p^{2}\right)^{2}} \int_{k} \frac{(\mathbf{q} / 4-\mathbf{p} / 2)^{2}-k^{2}}{(\mathbf{q} / 4-\mathbf{p} / 2+\mathbf{k})^{2}(\mathbf{q} / 4-\mathbf{p} / 2-\mathbf{k})^{2}}$

$\times \frac{(\mathbf{q} / 2-\mathbf{p})^{2}\left[(\mathbf{q} / 4-\mathbf{p} / 2)^{2}+k^{2}\right]-2[(\mathbf{q} \mathbf{k}) / 2-(\mathbf{p k})]^{2}}{i \omega / 2 \nu_{0}+(\mathbf{q} / 2+\mathbf{p})^{2} / 2+(\mathbf{q} / 2-\mathbf{p})^{2} / 4+k^{2}}$,

$(d)=-\frac{\lambda_{0}^{4} D_{0}^{2}}{32 \nu_{0}^{5}} \int_{p} \frac{q^{2} / 4-p^{2}}{(\mathbf{q} / 2-\mathbf{p})^{4}} \frac{q^{2}-2(\mathbf{q p})}{i \omega / 2 \nu_{0}+q^{2} / 4+p^{2}}$

$\times \int_{k} \frac{(\mathbf{q} / 4-\mathbf{p} / 2)^{2}-k^{2}}{3(\mathbf{q} / 2-\mathbf{p})^{2} / 4+k^{2}} \frac{(\mathbf{q} / 2-\mathbf{p})^{2}\left[(\mathbf{q} / 4-\mathbf{p} / 2)^{2}+k^{2}\right]-2[(\mathbf{q} \mathbf{k}) / 2-(\mathbf{p k})]^{2}}{(\mathbf{q} / 4-\mathbf{p} / 2+\mathbf{k})^{2}(\mathbf{q} / 4-\mathbf{p} / 2-\mathbf{k})^{2}}$

$\times\left[1+\frac{(\mathbf{q} / 2-\mathbf{p})^{2}}{i \omega / 2 \nu_{0}+(\mathbf{q} / 2+\mathbf{p})^{2} / 2+(\mathbf{q} / 2-\mathbf{p})^{2} / 4+k^{2}}\left(1+\frac{3(\mathbf{q} / 2-\mathbf{p})^{2} / 4+k^{2}}{i \omega / 2 \nu_{0}+q^{2} / 4+p^{2}}\right)\right]$,

$(e)=-\frac{\lambda_{0}^{4} D_{0}^{2}}{32 \nu_{0}^{5}} \int_{p} \frac{q^{2} / 4-p^{2}}{(\mathbf{q} / 2-\mathbf{p})^{4}} \frac{q^{2}-2(\mathbf{q p})}{i \omega / 2 \nu_{0}+q^{2} / 4+p^{2}}$

$\times \int_{k} \frac{(\mathbf{q} / 4-\mathbf{p} / 2)^{2}-k^{2}}{3(\mathbf{q} / 2-\mathbf{p})^{2} / 4+k^{2}} \frac{(\mathbf{q} / 2-\mathbf{p})^{2}\left[(\mathbf{q} / 4-\mathbf{p} / 2)^{2}+k^{2}\right]-2[(\mathbf{q k}) / 2-(\mathbf{p k})]^{2}}{(\mathbf{q} / 4-\mathbf{p} / 2+\mathbf{k})^{2}(\mathbf{q} / 4-\mathbf{p} / 2-\mathbf{k})^{2}}$, 


$$
\begin{aligned}
& (f)=\frac{\lambda_{0}^{4} D_{0}^{2}}{32 \nu_{0}^{5}} \int_{p} \frac{q^{2} / 4-p^{2}}{(\mathbf{q} / 2-\mathbf{p})^{2}} \frac{q^{2}-2(\mathbf{q p})}{i \omega / 2 \nu_{0}+q^{2} / 4+p^{2}} \int_{k} \frac{1}{3(\mathbf{q} / 2-\mathbf{p})^{2} / 4+k^{2}} \\
& \times \frac{\left[(\mathbf{q} / 4-\mathbf{p} / 2)^{2}-k^{2}\right]^{2}}{(\mathbf{q} / 4-\mathbf{p} / 2+\mathbf{k})^{2}(\mathbf{q} / 4-\mathbf{p} / 2-\mathbf{k})^{2}}\left(2+\frac{(\mathbf{q} / 2-\mathbf{p})^{2}}{i \omega / 2 \nu_{0}+(\mathbf{q} / 2+\mathbf{p})^{2} / 2+(\mathbf{q} / 2-\mathbf{p})^{2} / 4+k^{2}}\right), \\
& (g)=\frac{\lambda_{0}^{4} D_{0}^{2}}{8 \nu_{0}^{5}} \int_{p} \frac{q^{2}-2(\mathbf{q p})}{(\mathbf{q} / 2-\mathbf{p})^{2}\left(i \omega / 2 \nu_{0}+q^{2} / 4+p^{2}\right)} \int_{k} \frac{(\mathbf{q k}) / 2+(\mathbf{p k})}{(\mathbf{q} / 2-\mathbf{p}-\mathbf{k})^{2}} \\
& \times \frac{q^{2} / 4-(\mathbf{p}+\mathbf{k})^{2}}{i \omega / 2 \nu_{0}+q^{2} / 4+(\mathbf{p}+\mathbf{k})^{2}} \frac{(\mathbf{q k}) / 2-(\mathbf{p k})-(\mathbf{q} / 2-\mathbf{p})^{2}}{(\mathbf{q} / 2-\mathbf{p}-\mathbf{k})^{2}+(\mathbf{q} / 2-\mathbf{p})^{2}+k^{2}} \\
& \times\left(1+\frac{2(\mathbf{q} / 2-\mathbf{p})^{2}}{i \omega / \nu_{0}+(\mathbf{q} / 2-\mathbf{p}-\mathbf{k})^{2}+(\mathbf{q} / 2+\mathbf{p})^{2}+k^{2}}\right), \\
& (h)=\frac{\lambda_{0}^{4} D_{0}^{2}}{8 \nu_{0}^{5}} \int_{p} \frac{q^{2}+2(\mathbf{q p})}{(\mathbf{q} / 2+\mathbf{p})^{2}\left(i \omega / 2 \nu_{0}+q^{2} / 4+p^{2}\right)} \int_{k} \frac{(\mathbf{q k}) / 2+(\mathbf{p k})}{(\mathbf{q} / 2-\mathbf{p}-\mathbf{k})^{2}} \\
& \times \frac{q^{2} / 4-(\mathbf{p}+\mathbf{k})^{2}}{i \omega / 2 \nu_{0}+q^{2} / 4+(\mathbf{p}+\mathbf{k})^{2}} \frac{(\mathbf{q k}) / 2-(\mathbf{p k})-(\mathbf{q} / 2-\mathbf{p})^{2}}{i \omega / \nu_{0}+(\mathbf{q} / 2-\mathbf{p}-\mathbf{k})^{2}+(\mathbf{q} / 2+\mathbf{p})^{2}+k^{2}} \\
& (i)=-\frac{\lambda_{0}^{4} D_{0}^{2}}{8 \nu_{0}^{5}} \int_{p} \frac{q^{2}+2(\mathbf{q p})}{(\mathbf{q} / 2+\mathbf{p})^{2}\left(i \omega / 2 \nu_{0}+q^{2} / 4+p^{2}\right)} \int_{k} \frac{(\mathbf{q k})^{2} / 4-(\mathbf{p k})^{2}}{k^{2}} \\
& \times \frac{q^{2} / 4-(\mathbf{p}+\mathbf{k})^{2}}{i \omega / 2 \nu_{0}+q^{2} / 4+(\mathbf{p}+\mathbf{k})^{2}} \frac{1}{i \omega / \nu_{0}+(\mathbf{q} / 2-\mathbf{p}-\mathbf{k})^{2}+(\mathbf{q} / 2+\mathbf{p})^{2}+k^{2}} \\
& -\frac{\lambda_{0}^{4} D_{0}^{2}}{8 \nu_{0}^{5}} \int_{p} \frac{q^{2}-2(\mathbf{q p})}{(\mathbf{q} / 2-\mathbf{p})^{2}\left(i \omega / 2 \nu_{0}+q^{2} / 4+p^{2}\right)} \int_{k} \frac{\left[(\mathbf{q k})^{2} / 4-(\mathbf{p k})^{2}\right]\left[q^{2} / 4-(\mathbf{p}+\mathbf{k})^{2}\right]}{k^{2}\left[i \omega / 2 \nu_{0}+q^{2} / 4+(\mathbf{p}+\mathbf{k})^{2}\right]} \\
& \times \frac{1}{(\mathbf{q} / 2-\mathbf{p}-\mathbf{k})^{2}+(\mathbf{q} / 2-\mathbf{p})^{2}+k^{2}}\left(1+\frac{2(\mathbf{q} / 2-\mathbf{p})^{2}}{i \omega / \nu_{0}+(\mathbf{q} / 2-\mathbf{p}-\mathbf{k})^{2}+(\mathbf{q} / 2+\mathbf{p})^{2}+k^{2}}\right), \\
& (j)=\frac{\lambda_{0}^{4} D_{0}^{2}}{4 \nu_{0}^{5}} \int_{p} \frac{q^{2}-2(\mathbf{q p})}{(\mathbf{q} / 2-\mathbf{p})^{2}} \int_{k} \frac{(\mathbf{q k}) / 2+(\mathbf{p k})}{k^{2}(\mathbf{q} / 2-\mathbf{p}-\mathbf{k})^{2}} \frac{q^{2} / 4-(\mathbf{p}+\mathbf{k})^{2}}{i \omega / 2 \nu_{0}+q^{2} / 4+(\mathbf{p}+\mathbf{k})^{2}} \\
& \times \frac{(\mathbf{q k}) / 2-(\mathbf{p k})-k^{2}}{(\mathbf{q} / 2-\mathbf{p}-\mathbf{k})^{2}+(\mathbf{q} / 2-\mathbf{p})^{2}+k^{2}} \frac{(\mathbf{q} / 2-\mathbf{p})^{2}}{i \omega / \nu_{0}+(\mathbf{q} / 2-\mathbf{p}-\mathbf{k})^{2}+(\mathbf{q} / 2+\mathbf{p})^{2}+k^{2}} .
\end{aligned}
$$

Upon collecting the several contributions, these expressions simplify considerably. One finds that the vertex functions may be split into UV-singular and UV-regular parts according to $\Gamma_{\tilde{h} \tilde{h}}=\Gamma_{\tilde{h} \tilde{h}}^{\mathrm{reg}}+\Gamma_{\tilde{h} \tilde{h}}^{\text {sing }}$, and similarly $\Gamma_{\tilde{h} h}=\Gamma_{\tilde{h} h}^{\text {reg }}+\Gamma_{\tilde{h} h}^{\text {sing }}$. After some tedious but elementary algebra one arrives at the following explicit results:

$$
\begin{aligned}
\Gamma_{\tilde{h} \tilde{h}}(\mathbf{q}, \omega)^{\mathrm{reg}}= & \operatorname{Re}\left\{( i \omega + \nu _ { 0 } q ^ { 2 } ) \left[\frac{\lambda_{0}^{4} D_{0}^{3}}{4 \nu_{0}^{7}} \int_{\mathbf{p}} \frac{q^{2} / 4-p^{2}}{(\mathbf{q} / 2+\mathbf{p})^{2}} \frac{1}{i \omega / 2 \nu_{0}+q^{2} / 4+p^{2}}\right.\right. \\
& \times \int_{k} \frac{1}{i \omega / 2 \nu_{0}+q^{2} / 4+(\mathbf{p}+\mathbf{k})^{2}} \frac{(\mathbf{q k}) / 2+(\mathbf{p k})}{k^{2}(\mathbf{q} / 2-\mathbf{p}-\mathbf{k})^{2}} \frac{(\mathbf{q k}) / 2-(\mathbf{p k})-k^{2}}{i \omega / \nu_{0}+(\mathbf{q} / 2-\mathbf{p}-\mathbf{k})^{2}+(\mathbf{q} / 2+\mathbf{p})^{2}+k^{2}} \\
& +\frac{\lambda_{0}^{4} D_{0}^{3}}{\nu_{0}^{7}} \int_{p} \frac{q^{2} / 4-p^{2}}{(\mathbf{q} / 2+\mathbf{p})^{2}(\mathbf{q} / 2-\mathbf{p})^{2}} \int_{k} \frac{(\mathbf{q k}) / 2+(\mathbf{p k})}{i \omega / 2 \nu_{0}+q^{2} / 4+(\mathbf{p}+\mathbf{k})^{2}} \frac{1}{k^{2}(\mathbf{q} / 2-\mathbf{p}-\mathbf{k})^{2}} \\
& \times \frac{(\mathbf{q} / 2-\mathbf{p})^{2} k^{2}-[(\mathbf{q k}) / 2-(\mathbf{p k})]^{2}}{(\mathbf{q} / 2-\mathbf{p}-\mathbf{k})^{2}+(\mathbf{q} / 2-\mathbf{p})^{2}+k^{2}}\left(\operatorname{Re} \frac{1}{i \omega / 2 \nu_{0}+q^{2} / 4+p^{2}}\right. \\
& \left.\left.\left.+\frac{1}{i \omega / 2 \nu_{0}+q^{2} / 4+p^{2}} \frac{(\mathbf{q} / 2-\mathbf{p})^{2}}{i \omega / \nu_{0}+(\mathbf{q} / 2-\mathbf{p}-\mathbf{k})^{2}+(\mathbf{q} / 2+\mathbf{p})^{2}+k^{2}}\right)\right]\right\},
\end{aligned}
$$




$$
\begin{aligned}
\Gamma_{\tilde{h} \tilde{h}}(\mathbf{q}, \omega)^{\text {sing }}= & -2 D_{0}-\frac{\lambda_{0}^{2} D_{0}^{2}}{2 \nu_{0}^{3}} \operatorname{Re} \int_{p} \frac{\left(q^{2} / 4-p^{2}\right)^{2}}{(\mathbf{q} / 2+\mathbf{p})^{2}(\mathbf{q} / 2-\mathbf{p})^{2}} \frac{1}{i \omega / 2 \nu_{0}+q^{2} / 4+p^{2}} \\
& -\frac{\lambda_{0}^{4} D_{0}^{3}}{8 \nu_{0}^{6}} \operatorname{Re} \int_{p} \frac{\left(q^{2} / 4-p^{2}\right)^{2}}{(\mathbf{q} / 2+\mathbf{p})^{2}} \frac{1}{\left(i \omega / 2 \nu_{0}+q^{2} / 4+p^{2}\right)^{2}} \int_{k} \frac{(\mathbf{q} / 4-\mathbf{p} / 2)^{2}-k^{2}}{(\mathbf{q} / 4-\mathbf{p} / 2+\mathbf{k})^{2}(\mathbf{q} / 4-\mathbf{p} / 2-\mathbf{k})^{2}} \\
& -\frac{\lambda_{0}^{4} D_{0}^{3}}{4 \nu_{0}^{6}} \operatorname{Re} \int_{p} \frac{\left(q^{2} / 4-p^{2}\right)^{2}}{(\mathbf{q} / 2+\mathbf{p})^{2}(\mathbf{q} / 2-\mathbf{p})^{2}} \frac{1}{\left(i \omega / 2 \nu_{0}+q^{2} / 4+p^{2}\right)^{2}} \int_{k} \frac{(\mathbf{q} / 2-\mathbf{p})^{2} k^{2}-[(\mathbf{q k}) / 2-(\mathbf{p k})]^{2}}{(\mathbf{q} / 4-\mathbf{p} / 2+\mathbf{k})^{2}(\mathbf{q} / 4-\mathbf{p} / 2-\mathbf{k})^{2}} \\
& -\frac{\lambda_{0}^{4} D_{0}^{3}}{2 \nu_{0}^{6}} \operatorname{Re} \int_{p} \frac{\left(q^{2} / 4-p^{2}\right)^{2}}{(\mathbf{q} / 2+\mathbf{p})^{2}(\mathbf{q} / 2-\mathbf{p})^{4}} \frac{1}{i \omega / 2 \nu_{0}+q^{2} / 4+p^{2}} \\
& \times \int_{k} \frac{(\mathbf{q} / 2-\mathbf{p})^{2} k^{2}-[(\mathbf{q} \mathbf{k}) / 2-(\mathbf{p k})]^{2}}{(\mathbf{q} / 4-\mathbf{p} / 2+\mathbf{k})^{2}(\mathbf{q} / 4-\mathbf{p} / 2-\mathbf{k})^{2}},
\end{aligned}
$$

$$
\begin{aligned}
\Gamma_{\tilde{h} h}(\mathbf{q}, \omega)^{\mathbf{r e g}}= & -\left(i \omega+\nu_{0} q^{2}\right)\left[\frac{\lambda_{0}^{4} D_{0}^{2}}{8 \nu_{0}^{6}} \int_{p} \frac{q^{2}\left(q^{2} / 4+p^{2}\right)-2(\mathbf{q p})^{2}}{(\mathbf{q} / 2+\mathbf{p})^{2}\left(i \omega / 2 \nu_{0}+q^{2} / 4+p^{2}\right)}\right. \\
& \times \int_{k} \frac{1}{i \omega / 2 \nu_{0}+q^{2} / 4+(\mathbf{p}+\mathbf{k})^{2}} \frac{(\mathbf{q k}) / 2+(\mathbf{p k})}{k^{2}(\mathbf{q} / 2-\mathbf{p}-\mathbf{k})^{2}} \frac{(\mathbf{q k}) / 2-(\mathbf{p k})-k^{2}}{i \omega / \nu_{0}+(\mathbf{q} / 2-\mathbf{p}-\mathbf{k})^{2}+(\mathbf{q} / 2+\mathbf{p})^{2}+k^{2}} \\
& +\frac{\lambda_{0}^{4} D_{0}^{2}}{4 \nu_{0}^{6}} \int_{p} \frac{q^{2}\left(q^{2} / 4+p^{2}\right)-2(\mathbf{q p})^{2}}{(\mathbf{q} / 2+\mathbf{p})^{2}(\mathbf{q} / 2-\mathbf{p})^{2}\left(i \omega / 2 \nu_{0}+q^{2} / 4+p^{2}\right)} \int_{k} \frac{1}{i \omega / 2 \nu_{0}+q^{2} / 4+(\mathbf{p}+\mathbf{k})^{2}} \\
& \times \frac{(\mathbf{q k}) / 2+(\mathbf{p k})}{k^{2}(\mathbf{q} / 2-\mathbf{p}-\mathbf{k})^{2}} \frac{(\mathbf{q} / 2-\mathbf{p})^{2} k^{2}-[(\mathbf{q k}) / 2-(\mathbf{p k})]^{2}}{i\left(\nu_{0}+(\mathbf{q} / 2-\mathbf{p}-\mathbf{k})^{2}+(\mathbf{q} / 2+\mathbf{p})^{2}+k^{2}\right.} \\
& +\frac{\lambda_{0}^{4} D_{0}^{2}}{4 \nu_{0}^{6}} \int_{p} \frac{q^{2}-2(\mathbf{q} \mathbf{p})}{(\mathbf{q} / 2-\mathbf{p})^{2}} \int_{k} \frac{1}{i \omega / 2 \nu_{0}+q^{2} / 4+(\mathbf{p}+\mathbf{k})^{2}} \frac{(\mathbf{q} \mathbf{k}) / 2+(\mathbf{p k})}{k^{2}(\mathbf{q} / 2-\mathbf{p}-\mathbf{k})^{2}} \\
& \left.\times \frac{(\mathbf{q} / 2-\mathbf{p})^{2} k^{2}-[(\mathbf{q k}) / 2-(\mathbf{p k})]^{2}}{\left[(\mathbf{q} / 2-\mathbf{p}-\mathbf{k})^{2}+(\mathbf{q} / 2-\mathbf{p})^{2}+k^{2}\right]\left[i \omega / \nu_{0}+(\mathbf{q} / 2-\mathbf{p}-\mathbf{k})^{2}+(\mathbf{q} / 2+\mathbf{p})^{2}+k^{2}\right]}\right]
\end{aligned}
$$

$$
\begin{aligned}
\Gamma_{\tilde{h} h}(\mathbf{q}, \omega)^{\text {sing }}= & i \omega+\nu_{0} q^{2}+\frac{\lambda_{0}^{2} D_{0}}{4 \nu_{0}^{2}} \int_{p} \frac{q^{2} / 4-p^{2}}{(\mathbf{q} / 2+\mathbf{p})^{2}(\mathbf{q} / 2-\mathbf{p})^{2}} \frac{q^{2}\left(q^{2} / 4+p^{2}\right)-2(\mathbf{q p})^{2}}{i \omega / 2 \nu_{0}+q^{2} / 4+p^{2}} \\
& +\frac{\lambda_{0}^{4} D_{0}^{2}}{16 \nu_{0}^{5}} \int_{p} \frac{q^{2} / 4-p^{2}}{(\mathbf{q} / 2+\mathbf{p})^{2}} \frac{q^{2}\left(q^{2} / 4+p^{2}\right)-2(\mathbf{q} \mathbf{p})^{2}}{\left(i \omega / 2 \nu_{0}+q^{2} / 4+p^{2}\right)^{2}} \int_{k} \frac{(\mathbf{q} / 4-\mathbf{p} / 2)^{2}-k^{2}}{(\mathbf{q} / 4-\mathbf{p} / 2+\mathbf{k})^{2}(\mathbf{q} / 4-\mathbf{p} / 2-\mathbf{k})^{2}} \\
& +\frac{\lambda_{0}^{4} D_{0}^{2}}{8 \nu_{0}^{5}} \int_{p} \frac{q^{2} / 4-p^{2}}{(\mathbf{q} / 2+\mathbf{p})^{2}(\mathbf{q} / 2-\mathbf{p})^{2}} \frac{q^{2}\left(q^{2} / 4+p^{2}\right)-2(\mathbf{q p})^{2}}{\left(i \omega / 2 \nu_{0}+q^{2} / 4+p^{2}\right)^{2}} \int_{k} \frac{(\mathbf{q} / 2-\mathbf{p})^{2} k^{2}-[(\mathbf{q k}) / 2-(\mathbf{p k})]^{2}}{(\mathbf{q}-\mathbf{p} / 2+\mathbf{k})^{2}(\mathbf{q} / 4-\mathbf{p} / 2-\mathbf{k})^{2}} \\
& +\frac{\lambda_{0}^{4} D_{0}^{2}}{8 \nu_{0}^{5}} \int_{p} \frac{q^{2} / 4-p^{2}}{(\mathbf{q} / 2-\mathbf{p})^{4}} \frac{q^{2}-2(\mathbf{q} \mathbf{p})}{i \omega / 2 \nu_{0}+q^{2} / 4+p^{2}} \int_{k} \frac{(\mathbf{q} / 2-\mathbf{p})^{2} k^{2}-[(\mathbf{q} \mathbf{k}) / 2-(\mathbf{p k})]^{2}}{(\mathbf{q} / 4-\mathbf{p} / 2+\mathbf{k})^{2}(\mathbf{q} / 4-\mathbf{p} / 2-\mathbf{k})^{2}} \\
& -\frac{\lambda_{0}^{4} D_{0}^{2}}{16 \nu_{0}^{5}} \int_{p} \frac{q^{2} / 4-p^{2}}{(\mathbf{q} / 2-\mathbf{p})^{2}} \frac{q^{2}-2(\mathbf{q p})}{i \omega / 2 \nu_{0}+q^{2} / 4+p^{2}} \\
& \times \int_{k} \frac{(\mathbf{q} / 2-\mathbf{p})^{2} k^{2}-[(\mathbf{q k}) / 2-(\mathbf{p k})]^{2}}{(\mathbf{q} / 4-\mathbf{p} / 2+\mathbf{k})^{2}(\mathbf{q} / 4-\mathbf{p} / 2-\mathbf{k})^{2}\left[3(\mathbf{q} / 2-\mathbf{p})^{2} / 4+k^{2}\right]}
\end{aligned}
$$

A powerful check to these lengthy calculations is the investigation of the situation at $d=1$, where the above expressions simplify considerably. Using some elementary algebra again, one finds at $d=1$

$$
\frac{1}{\nu_{0} q^{2}} \operatorname{Re}_{\tilde{h} h}(q, \omega)=-\frac{1}{2 D_{0}} \Gamma_{\tilde{h} \tilde{h}}(q, \omega),
$$

which ensures the validity of the fluctuation-dissipation theorem, for both renormalized momentum- and frequencydependent quantities $\nu(q, \omega)$ and $D(q, \omega)$, defined as the left- and right-hand sides of Eq. (A20), respectively, coincide. The explicit result reads

$$
\begin{aligned}
\nu(q, \omega)= & \nu_{0}\left[1+\frac{\lambda_{0}^{2} D_{0}}{4 \nu_{0}^{3}} \operatorname{Re} \int_{p} \frac{1}{i \omega / 2 \nu_{0}+q^{2} / 4+p^{2}}+\frac{\lambda_{0}^{4} D_{0}^{2}}{16 \nu_{0}^{6}} \operatorname{Re} \int_{p} \frac{(q / 2-p)^{2}}{\left(i \omega / 2 \nu_{0}+q^{2} / 4+p^{2}\right)^{2}} \int_{k} \frac{1}{k(q / 2-p-k)}\right. \\
& -\frac{\lambda_{0}^{4} D_{0}^{2}}{8 \nu_{0}^{6}} \operatorname{Re}\left(\left(i \omega / \nu_{0} q^{2}\right) \int_{p} \frac{q / 2-p}{i \omega / 2 \nu_{0}+q^{2} / 4+p^{2}} \int_{k} \frac{1}{i \omega / 2 \nu_{0}+q^{2} / 4+(p+k)^{2}}\right. \\
& \left.\left.\times \frac{1}{q / 2-p-k} \frac{1}{i \omega / \nu_{0}+(q / 2-p-k)^{2}+(q / 2+p)^{2}+k^{2}}\right)\right]
\end{aligned}
$$


which already is a useful and interesting result of the two-loop calculation on its own standing.

We now return to the general $d$-dimensional case. In evaluating the UV-singular contributions, one has to be careful to choose a NP where either $q$ or $\omega$ is finite in order not to interfere with the IR singularities, which would show up as poles in $1 /(d-2)$, too. A convenient choice for the NP is $q=0, i \omega / 2 \nu=\mu^{2}$; thus we find

$$
\begin{aligned}
& \left.\Gamma_{\tilde{h} \tilde{h}}(\mathbf{q}, \omega)^{\text {sing }}\right|_{\mathrm{NP}}=-2 D_{0}\left[1+g_{0} \int_{p} \frac{1}{\mu^{2} Z_{\nu}+p^{2}}+g_{0}^{2} \int_{p} \frac{p^{2}}{\left(\mu^{2}+p^{2}\right)^{2}} \int_{k} \frac{p^{2} / 4-k^{2}}{(\mathbf{p} / 2+\mathbf{k})^{2}(\mathbf{p} / 2-\mathbf{k})^{2}}\right. \\
& +2 g_{0}^{2} \int_{p} \frac{1}{\left(\mu^{2}+p^{2}\right)^{2}} \int_{k} \frac{p^{2} k^{2}-(\mathbf{p k})^{2}}{(\mathbf{p} / 2+\mathbf{k})^{2}(\mathbf{p} / 2-\mathbf{k})^{2}} \\
& \left.+4 g_{0}^{2} \int_{p} \frac{1}{p^{2}\left(\mu^{2}+p^{2}\right)} \int_{k} \frac{p^{2} k^{2}-(\mathbf{p k})^{2}}{(\mathbf{p} / 2+\mathbf{k})^{2}(\mathbf{p} / 2-\mathbf{k})^{2}}+O\left(g_{0}^{3}\right)\right] \\
& \left.\frac{\partial}{\partial q^{2}} \Gamma_{\tilde{h} h}(\mathbf{q}, \omega)^{\text {sing }}\right|_{\mathrm{NP}}=\nu_{0}\left[1-\frac{d-2}{d} g_{0} \int_{p} \frac{1}{\mu^{2} Z_{\nu}+p^{2}}-\frac{d-2}{d} g_{0}^{2} \int_{p} \frac{p^{2}}{\left(\mu^{2}+p^{2}\right)^{2}} \int_{k} \frac{p^{2} / 4-k^{2}}{(\mathbf{p} / 2+\mathbf{k})^{2}(\mathbf{p} / 2-\mathbf{k})^{2}}\right. \\
& -2 \frac{d-2}{d} g_{0}^{2} \int_{p} \frac{1}{\left(\mu^{2}+p^{2}\right)^{2}} \int_{k} \frac{p^{2} k^{2}-(\mathbf{p k})^{2}}{(\mathbf{p} / 2+\mathbf{k})^{2}(\mathbf{p} / 2-\mathbf{k})^{2}} \\
& -2 \frac{d-2}{d} g_{0}^{2} \int_{p} \frac{1}{p^{2}\left(\mu^{2}+p^{2}\right)} \int_{k} \frac{p^{2} k^{2}-(\mathbf{p k})^{2}}{(\mathbf{p} / 2+\mathbf{k})^{2}(\mathbf{p} / 2-\mathbf{k})^{2}} \\
& +g_{0}^{2} \int_{p} \frac{1}{\mu^{2}+p^{2}} \int_{k} \frac{p^{2} k^{2}-(\mathbf{p k})^{2}}{(\mathbf{p} / 2+\mathbf{k})^{2}(\mathbf{p} / 2-\mathbf{k})^{2}\left(3 p^{2} / 4+k^{2}\right)} \\
& +\frac{2}{d} g_{0}^{2} \int_{p} \frac{1}{\mu^{2}+p^{2}} \int_{k} \frac{\left[p^{2} k^{2}-(\mathbf{p k})^{2}\right] k^{2}}{(\mathbf{p} / 2+\mathbf{k})^{2}(\mathbf{p} / 2-\mathbf{k})^{2}\left(3 p^{2} / 4+k^{2}\right)^{2}} \\
& \left.+\frac{4}{d} g_{0}^{2} \int_{p} \frac{1}{p^{2}\left(\mu^{2}+p^{2}\right)} \int_{k} \frac{\left(p^{2} / 4-k^{2}\right)\left[p^{2} k^{2}-(\mathbf{p k})^{2}\right](\mathbf{p k})^{2}}{(\mathbf{p} / 2+\mathbf{k})^{4}(\mathbf{p} / 2-\mathbf{k})^{4}\left(3 p^{2} / 4+k^{2}\right)}+O\left(g_{0}^{3}\right)\right] \text {. }
\end{aligned}
$$

For the derivation of the latter expression, the following relations have proven very useful:

$$
\begin{gathered}
\int_{p}(\mathbf{q p}) f(\mathbf{p})=\frac{q^{2}}{d} \int_{p} p^{2} f(p) \\
\int_{p} \int_{k}(\mathbf{q p})(\mathbf{q} \mathbf{k})(\mathbf{p k}) f(\mathbf{p}, \mathbf{k})=\frac{q^{2}}{d} \int_{p} \int_{k}(\mathbf{p k})^{2} f(\mathbf{p}, \mathbf{k}) .
\end{gathered}
$$

\section{Integrals in dimensional regularization}

In this section we list the results for the above integrals, as obtained from the dimensional regularization scheme $[23,24]$ :

$$
\begin{aligned}
& \int_{p} \frac{1}{\mu^{2}+p^{2}}=-\frac{C_{d} \mu^{\epsilon}}{\epsilon} \\
& \int_{p} \frac{p^{2}}{\left(\mu^{2}+p^{2}\right)^{2}} \int_{k} \frac{p^{2} / 4-k^{2}}{(\mathbf{p} / 2+\mathbf{k})^{2}(\mathbf{p} / 2-\mathbf{k})^{2}}=-(d-1) \frac{C_{d}^{2} \mu^{2 \epsilon}}{2 \epsilon^{2}} \frac{\Gamma(1-\epsilon) \Gamma(1+\epsilon / 2)}{\Gamma(1-\epsilon / 2)} \\
& \int_{p} \frac{1}{\left(\mu^{2}+p^{2}\right)^{2}} \int_{k} \frac{p^{2} k^{2}-(\mathbf{p k})^{2}}{(\mathbf{p} / 2+\mathbf{k})^{2}(\mathbf{p} / 2-\mathbf{k})^{2}}=(d-1) \frac{C_{d}^{2} \mu^{2 \epsilon}}{4 \epsilon^{2}} \frac{\Gamma(1-\epsilon) \Gamma(1+\epsilon / 2)}{\Gamma(1-\epsilon / 2)} \\
& \int_{p} \frac{1}{p^{2}\left(\mu^{2}+p^{2}\right)} \int_{k} \frac{p^{2} k^{2}-(\mathbf{p k})^{2}}{(\mathbf{p} / 2+\mathbf{k})^{2}(\mathbf{p} / 2-\mathbf{k})^{2}}=(d-1) \frac{C_{d}^{2} \mu^{2 \epsilon}}{4 \epsilon^{2}(1+\epsilon)} \frac{\Gamma(1-\epsilon) \Gamma(1+\epsilon / 2)}{\Gamma(1-\epsilon / 2)} \\
& \int_{p} \frac{1}{\mu^{2}+p^{2}} \int_{k} \frac{p^{2} k^{2}-(\mathbf{p k})^{2}}{(\mathbf{p} / 2+\mathbf{k})^{2}(\mathbf{p} / 2-\mathbf{k})^{2}\left(3 p^{2} / 4+k^{2}\right)}=-(d-1) \frac{C_{d}^{2} \mu^{2 \epsilon}}{8 \epsilon} \frac{\Gamma(1-\epsilon) \Gamma(1+\epsilon)}{\Gamma(1-\epsilon / 2) \Gamma(1+\epsilon / 2)} I_{00}(d),
\end{aligned}
$$




$$
\begin{aligned}
\int_{p} \frac{1}{\mu^{2}+p^{2}} \int_{k} & \frac{\left[p^{2} k^{2}-(\mathbf{p k})^{2}\right] k^{2}}{(\mathbf{p} / 2+\mathbf{k})^{2}(\mathbf{p} / 2-\mathbf{k})^{2}\left(3 p^{2} / 4+k^{2}\right)^{2}} \\
= & -(d-1) \frac{C_{d}^{2} \mu^{2 \epsilon}}{16 \epsilon} \frac{\Gamma(1-\epsilon) \Gamma(1+\epsilon)}{\Gamma(1-\epsilon / 2) \Gamma(1+\epsilon / 2)}\left[(d+\epsilon) I_{10}(d)+\frac{2-\epsilon}{4} I_{11}(d)+\frac{2-\epsilon}{2} I_{21}(d)\right] \\
\int_{p} \frac{1}{p^{2}\left(\mu^{2}+p^{2}\right)} \int_{k} & \frac{\left(p^{2} / 4-k^{2}\right)\left[p^{2} k^{2}-(\mathbf{p k})^{2}\right](\mathbf{p k})^{2}}{(\mathbf{p} / 2+\mathbf{k})^{4}(\mathbf{p} / 2-\mathbf{k})^{4}\left(3 p^{2} / 4+k^{2}\right)} \\
= & (d-1) \frac{(2-\epsilon) C_{d}^{2} \mu^{2 \epsilon}}{32 \epsilon} \frac{\Gamma(1-\epsilon) \Gamma(1+\epsilon)}{\Gamma(1-\epsilon / 2) \Gamma(1+\epsilon / 2)}\left[(3-\epsilon) I_{00}(d)-\frac{10-3 \epsilon}{2} I_{11}(d)\right. \\
& +\frac{3-\epsilon}{4} I_{21}(d)+\frac{7(4-\epsilon)}{16} I_{22}(d)-\frac{4-\epsilon}{8} I_{32}(d)+\frac{1}{2} \int_{0}^{1} \frac{x(1-x)}{\left(1+x-x^{2}\right)^{2-\epsilon / 2}} d x \\
& -\frac{4-\epsilon}{4}\left[\tilde{I}_{01}(d)-\tilde{I}_{12}(d)\right]+(d-1) \frac{C_{d}^{2} \mu^{2 \epsilon}}{16 \epsilon^{2}} \frac{\Gamma(1-\epsilon) \Gamma(1+\epsilon / 2)}{\Gamma(1-\epsilon / 2)}\left(\frac{2}{1+\epsilon}-(2-\epsilon)\right)
\end{aligned}
$$

Here $C_{d}=\Gamma(2-d / 2) / 2^{d-1} \pi^{d / 2}$ is a geometry factor, while

$$
I_{r s}(d)=\int_{0}^{1} \int_{0}^{1} \frac{y(1-y)^{r}}{\left[x(1-x) y^{2}+(1-y)(3+y) / 4\right]^{s+2-d / 2}} d x d y
$$

and

$$
\tilde{I}_{r s}(d)=I_{r s}(d)-\int_{0}^{1} \int_{0}^{1} \frac{(1-y)^{r}}{[x(1-x)+1-y]^{s+2-d / 2}} d x d y
$$

are parameter integrals emerging upon the use of Feynman parametrization. Finally, we note that

$$
\begin{gathered}
\frac{\Gamma(1-\epsilon) \Gamma(1+\epsilon / 2)}{\Gamma(1-\epsilon / 2)}=1+O\left(\epsilon^{2}\right), \\
\frac{\Gamma(1-\epsilon) \Gamma(1+\epsilon)}{\Gamma(1-\epsilon / 2) \Gamma(1+\epsilon / 2)}=1+O\left(\epsilon^{2}\right),
\end{gathered}
$$

and

$$
\int_{0}^{1} \frac{x(1-x)}{\left(1+x-x^{2}\right)^{2}} d x=\frac{6}{5 \sqrt{5}} \ln \frac{\sqrt{5}+1}{\sqrt{5}-1}-\frac{2}{5}
$$

are to be used when performing the $\epsilon$ expansion leading to the results for the $\mathrm{Z}$ factors. Note also that at $d=2$

$$
\begin{array}{r}
8 I_{00}(2)-4 I_{10}(2)-21 I_{11}(2)+I_{21}(2)+7 I_{22}(2)-2 I_{32}(2)-4 \tilde{I}_{01}(2)+4 \tilde{I}_{12}(2)+\frac{12}{5 \sqrt{5}} \ln \frac{\sqrt{5}+1}{\sqrt{5}-1}-\frac{4}{5}+4 \\
=8-F_{\nu}(2)=0 .
\end{array}
$$

[1] For a recent review, see J. Krug and H. Spohn, in Solids Far From Equilibrium: Growth, Morphology and Defects, edited by C. Godriche (Cambridge University Press, Cambridge, 1992).

[2] See, e.g., H. van Beijeren and I. Nolden, in Structure and Dynamics of Surfaces II, edited by W. Schommers and P. von Blakenhagen (Springer-Verlag, Berlin, 1987); see also T. Hwa, M. Kardar, and M. Paczuski, Phys. Rev. Lett. 66, 441 (1991), for a discussion of the nonequilibrium aspect.

[3] M. Kardar, G. Parisi, and Y.-C. Zhang, Phys. Rev. Lett. 56, 889 (1986); E. Medina, T. Hwa, M. Kardar, and Y.-
C. Zhang, Phys. Rev. A 39, 3053 (1989).

[4] D. Forster, D. R. Nelson, and M. J. Stephen, Phys. Rev. A 16, 732 (1977).

[5] H. van Beijeren, R. Kutner, and H. Spohn, Phys. Rev. Lett. 54, 2026 (1985); H. K. Janssen and B. Schmittmann, Z. Phys. B 63, 517 (1986).

[6] J. Krug and H. Spohn, Europhys. Lett. 8, 219 (1989).

[7] V. S. L'vov and I. Procaccia, Phys. Rev. Lett. 69, 3543 (1992); I. Proccacia, M. H. Jensen, V. S. L'vov, K. Sneppen, and R. Zeitak, Phys. Rev. A 46, 3220 (1993).

[8] M. Kardar and Y.-C. Zhang, Phys. Rev. Lett. 58, 2087 (1987). 
[9] S. Zalesky, Physica D 34, 417 (1989) and references therein.

[10] T. Hwa, Phys. Rev. Lett. 69, 1552 (1992).

[11] D. A. Huse, C. L. Henley, and D. S. Fisher, Phys. Rev. Lett. 55, 2924 (1985); M. Kardar and Y.-C. Zhang, ibid. 58, 2087 (1987).

[12] For a recent review, see D. S. Fisher and D. A. Huse, Phys. Rev. B 43, 10728 (1991); see also G. Parisi, J. Phys. (Paris) 51, 1595 (1990); M. Mezard, ibid. 51, 1831 (1990).

[13] S. F. Edwards and D. R. Wilkinson, Proc. R. Soc. London Ser. A 381, 17 (1982).

[14] B. M. Forrest and L.-H. Tang, Phys. Rev. Lett. 64, 1405 (1990); T. Ala-Nissila, T. Hjelt, and J. M. Kosterlitz, Europhys. Lett. 19, 1 (1992); T. Ala-Nissila, T. Hjelt, J. M. Kosterlitz, and O. Venäläinen, J. Stat. Phys. 72, 207 (1993).

[15] D. E. Wolf and J. Kertesz, Europhys. Lett. 4, 651 (1987).

[16] J. M. Kim and J. M. Kosterlitz, Phys. Rev. Lett. 62, 2289 (1989).

[17] Strictly speaking, the one-loop perturbation theory in Refs. $[3,4]$ gives a strong-coupling fixed point for $d<3 / 2$ only. But this is an artifact of the one-loop approximation and will be corrected by the two-loop calculations presented in this paper.

[18] C. A. Doty and J. M. Kosterlitz, Phys. Rev. Lett. 69, 1979 (1992).

[19] L.-H. Tang, T. Nattermann, and B. M. Forrest, Phys. Rev. Lett. 65, 2422 (1990).

[20] T. Nattermann and L.-H. Tang, Phys. Rev. B 45, 7156 (1992).

[21] H. K. Janssen, Z. Phys. B 23, 377 (1976).

[22] R. Baussch, H. K. Janssen, and H. Wagner, Z. Phys. B 24, 113 (1976).

[23] G. 't Hooft and M. Veltman, Nucl. Phys. B 44, 189 (1972).

[24] D. J. Amit, Field Theory, the Renormalization Group, and Critical Phenomena, 2nd ed. (World Scientific, Singapore, 1984).

[25] J. Zinn-Justin, Quantum Field Theory and Critical Phenomena (Clarendon Press, Oxford, 1989).

[26] A. M. Polyakov, Phys. Lett. 59B, 79 (1975); E. Brézin and J. Zinn-Justin, Phys. Rev. B 14, 3110 (1976); D. R. Nelson and R. A. Pelcovits, ibid. 16, 2191 (1977); see also Ref. [24], Chap. II.6, and Ref. [25], Chap. 27.

[27] T. Sun and M. Plischke, Phys. Rev. E 49, 5046 (1994).

[28] C. de Dominicis, Nuovo Cimento Lett. 12, 567 (1975); J. Phys. (Paris) Colloq. 37, C1-247 (1976).

[29] P. C. Martin, E. D. Siggia, and H. H. Rose, Phys. Rev. A 8, 423 (1973).

[30] U. Deker and F. Haake, Phys. Rev. A 11, 2043 (1975).

[31] R. Graham, in Quantum Statistics in Optics and SolidState Physics, Springer Tracts in Modern Physics Vol. 66 (Springer-Verlag, Berlin, 1973).

[32] For a review of the application of mode-coupling theories to dynamic critical phenomena see, e.g., K. Kawasaki, in Phase Transitions and Critical Phenomena, edited by C. Domb and M. S. Green (Academic Press, New York, 1976), Vol. 5a.

[33] J. Krug, Phys. Rev. A 36, 5465 (1987).

[34] T. Hwa and E. Frey, Phys. Rev. A 44, R7873 (1991).

[35] J. P. Bouchaud and M. E. Cates, Phys. Rev. B 47, R1455
(1993); 48, 635(E) (1993).

[36] J. P. Doherty, M. A. Moore, J. M. Kim, and A. J. Bray, Phys. Rev. Lett. 72, 2041 (1994).

[37] E. Frey and H. Schinz (unpublished): We have repeated the numerical calculations of Refs. $[35,36]$ and find agreement with the results of Ref. [36], but disagree with the upper critical dimension found in Ref. [35].

[38] Y. Tu (unpublished).

[39] Sun and Plischke [27], however, obviously do not agree here. Instead they claim a singular contribution to $\Gamma_{\tilde{h} h}(0, \omega)$. In our opinion it is impossible to have such singular contributions for the following reasons. The identities in Sec. IIC are exact relations, which follow from Galilean invariance and the diffusive dynamics of the Burgers-KPZ equation. Those exact relations restrict the number of independent renormalization factors, as is well known from many other models in critical dynamics (see, e.g., Ref. [22]). In the dynamic functional for the BurgersKPZ equation there are only four terms, which can be renormalized by the introduction of the same number of counter terms or (equally well) by the same number of renormalization factors. Hence, if the theory is renormalizable, the maximum number of renormalization factors is four. We have introduced three, one for the noise amplitude, surface tension, and nonlinearity, respectively. In order to be complete, we could have introduced one more $Z$ factor to account for possible renormalizations of the $i \omega$ term in the dynamic functional. The way to do that is to some extent arbitrary. One choice would be to introduce an additional parameter in the $i \omega$ term; another choice is to introduce a renormalization factor for the fields $h$ and $\tilde{h}$. Whatever the choice would be, the exact relation in Sec. II C, following from the diffusive behavior of the Burgers-KPZ equation, implies that the corresponding $Z$ factor equals 1 . Additionally, the Galilean invariance yields that the nonlinearity does not renormalize. Hence we are left with two nontrivial renormalization factors.

[40] S. K. Ma and G. F. Mazenko, Phys. Rev. B 11, 4077 (1975).

[41] K. G. Wilson and J. Kogut, Phys. Rep. 12 C, 75 (1974).

[42] C. Itzykson and J.-M. Drouffe, Statistical Field Theory (Cambridge University Press, Cambridge, 1989), Vol. 1.

[43] Sun and Plischke [27] recover the FDT at $d=1$ by considering the one-dimensional case separately. Then they proceed with a $2-\epsilon$ expansion in the framework of which one would not expect the FDT to hold when one extrapolates to $\epsilon=1$. Note that we do not have to treat the one-dimensional case separately. Rather it comes out quite naturally, if one makes a clear distinction between $1 / \epsilon$ poles (UV singularities) and pure geometrical factors. The validity of the FDT is then a quite powerful check of the method employed.

[44] B. Derrida and H. Spohn, J. Stat. Phys. 51, 817 (1988).

[45] J. Cook and B. Derrida, Europhys. Lett. 10, 195 (1989); J. Phys. A 23, 1523 (1990).

[46] J. Z. Imbrie and T. Spencer, J. Stat. Phys. 52, 609 (1988).

[47] E. Frey, T. Hwa, and U. C. Täuber (unpublished).

[48] There are several diagrams shown in the work by Sun and Plischke [27] that violate causality; however, they are assigned a zero value eventually; see the Appendix of Ref. [27]. 